\title{
Feedback Coding Schemes for the Broadcast Channel with Mutual Secrecy Requirement at the Receivers
}

\author{
Bin Dai, Member, IEEE, Linman Yu, Xuxun Liu, Member, IEEE, and Zheng Ma, Member, IEEE
}

\begin{abstract}
The broadcast channel with mutual secrecy requirement at the receivers (BC-MSR-R) is a basic model characterizing the physical layer security (PLS) in the down-link of the wireless communication systems, where one transmitter sends two independent messages to two receivers via a broadcast channel, and each receiver can successfully decode his/her intended message and wishes to overhear the other one's message. This paper studies how to enhance the already existing secrecy rate region of the BC-MSR-R via receivers' feedback. Specifically, we propose two feedback strategies for the BC-MSR-R, where one uses the feedback to generate pure secret keys protecting the transmitted messages, and the other uses the feedback to generate not only keys but also cooperative messages helping the receivers to improve their decoding performance. Different inner bounds on the secrecy capacity region of the BC-MSR-R with noiseless feedback are constructed according to different feedback strategies, and these bounds are further illustrated by a Dueck-type example.
\end{abstract}

Index Terms-Broadcast channel, confidential message, feedback, secrecy capacity region.

\section{INTRODUCTION}

$\mathbf{S}$ ECURE communication over channels was first studied in the pioneering work [1], where a transmitter broadcasts its secret message to a legitimate receiver and an eavesdropper via a degraded broadcast channel, and perfect secrecy is achieved if the information leakage to the eavesdropper vanishes as the codeword length tends to infinity. Subsequently, reference [2] generalized the model studied in [1] by considering a general (not degraded) broadcast channel and the transmission of a common message which can be decoded at both the

Copyright (c) 2013 IEEE. Personal use of this material is permitted. However, permission to use this material for any other purposes must be obtained from the IEEE by sending a request to pubs-permissions@ieee.org

Manuscript received October 7, 2018; revised January 8, 2019, April 4, 2019, June 12, 2019; accepted June 15, 2019. This work was supported by the National Natural Science Foundation of China under Grants 61671391, U1734209, 61571373, the EU Marie Sklodowska-Curie individual Fellowship under Grant 796426, the China Scholarship Council (file No. 201807005013), and the 111 Project No.111-2-14. The editor coordinating the review of this manuscript and approving it for publication was Dr. Lawrence Ong.

B. Dai is with the School of Information Science and Technology, Southwest JiaoTong University, Chengdu 610031, China, e-mail: daibin@home.swjtu.edu.cn.

L. Yu is with the School of Economics and Management, Chengdu Textile College, Chengdu 611731, China, email: yulinmanylm@163.com.

$\mathrm{X}$. Liu is with the School of Electronic and Information Engineering, South China University of Technology, Guangzhou 510641, China, email: liuxuxun@scut.edu.cn.

Z. Ma is with the School of Electrical Engineering and Computer Science, Royal Institute of Technology (KTH), SE-10044, Stockholm, Sweden, e-mail: zma@kth.se. legitimate receiver and the eavesdropper. The follow-up studies of [1]-[2] include transmission of two independent messages respectively to two receivers over a general broadcast channel (the broadcast channel with mutual secrecy requirement at the receivers) [3]-[4], one transmitter broadcasts a secret message to multiple legitimate receivers and one eavesdropper (wiretap broadcast channel) [5]-[6], two transmitters send their secret messages to a legitimate receiver and an eavesdropper via a multiple-access channel (multiple-access wiretap channel) [7][8], multiple-access channel with mutual secrecy requirement at the transmitters [13], broadcast channel with relay [9]-[12], the two-way channel with an eavesdropper [14]-[15], and the interference channel with mutual secrecy requirement at the receivers [3].

In recent years, artificial noise aided cooperative jamming [16]-[18] has been shown to be a useful tool to enhance the achievable secrecy rates (reliable transmission rates subjec$\mathrm{t}$ to perfect secrecy constraint) of communication systems. However, we should note that in some circumstances, such as Internet of Things (IoT), the artificial noise aided cooperative jamming is not a good choice due to the energy constraint of the devices [19], and the channel feedback is another way to enhance the secrecy rates for such circumstances. The effect of channel feedback on the PLS of the communication systems was initially studied in [20], where the pioneering work on the wiretap channel [1] was re-visited by considering the situation that the legitimate receiver's received channel output is sent back to the transmitter through an additional noiseless feedback channel. Since the legitimate receiver's channel output is perfectly known by the transmitter and is not known by the eavesdropper, it can be used to generate secret key protecting the transmitted message. Combining the above idea of generating secret key from the feedback with the random binning scheme for the wiretap channel [1], [20] proposed a coding scheme which splits the transmitted message into two parts, where one is encoded along the lines of the random binning scheme for the wiretap channel, and the other is encrypted by the secret key. Compared with the secrecy capacity (i.e., channel capacity with perfect secrecy constraint) of the wiretap channel [1], it is easy to see that the part of message encrypted by the key leads to the fact that the channel output feedback increases the secrecy capacity of the wiretap channel.

Here note that in [20], the feedback channel is only used to send the legitimate receiver's channel output, and what happens when the feedback channel can be used to transmit 
anything as the legitimate parties wish? [21] studied this case and pointed out that the best choice of the legitimate parties is to send pure random bits (secret key) over the feedback channel. Assuming that the feedback channel is rate limited (i.e., the rate of the secret key is upper bounded by $R_{f}$ ), and using a coding scheme in the same way as that in [20], [21] showed that sending pure secret key is better than sending legitimate receiver's channel output if $R_{f}$ is larger than the key rate in [20], and vice versa. The work of [20] and [21] indicates that there is no difference between sending pure secret key and sending legitimate receiver's channel output, and the main purpose of the feedback is to allow the legitimate receiver and the transmitter to share the secret key. In recent years, the above secret key based feedback coding scheme has been widely used in communication systems with feedback. To be specific, for the communication channels with legitimate receiver's channel output feedback, [24]-[26] studied the PLS of the channels with memory or memoryless states and legitimate receiver's channel output feedback, and proposed variations of the secret key based feedback coding scheme in [20]. For the communication systems with feedback channels directly transmitting pure secret keys, [22] extended the work of [21] to a broadcast situation, where two legitimate receivers of the broadcast channel independently send their secret keys to the transmitter via two noiseless feedback channels, and these keys help to increase the achievable secrecy rate region of the broadcast wiretap channel [5]. [23] introduced memoryless channel state into the work of [21], and showed that the transmitted message can be protected by two keys, where one is from the feedback channel, and the other is generated by the channel state.

Very recently, [27] showed that for the PLS of communication systems with channel output feedback, a better usage of the feedback channel output is to generate not only key but also cooperative message from it, and this cooperative message helps to improve the legitimate receiver's decoding performance. [27] proved that for the wiretap channel with channel output feedback, this new feedback scheme performs better than the widely used secret key based feedback scheme. Moreover, [28] and [29] showed that the classical SchalkwijkKailath (SK) feedback scheme for the Gaussian channel [30] achieves the secrecy capacity of the Gaussian wiretap channel with channel output feedback, and it equals the capacity of the same channel model without the secrecy constraint. However, we should notice that the results of [28] and [29] only work in the Gaussian case.

In wireless communication systems, each receiver can decode his/her intended message and overhear other ones' intended messages due to the broadcast nature of wireless communication. This communication scenario can be modeled by the broadcast channel with mutual secrecy requirement at the receivers (BC-MSR-R) [3]-[4], which is now a basic model for the PLS in the down-link of wireless communication systems. As a natural extension of the previous works [20][27], understanding the feedback effects on the BC-MSR-R is of great interest in the PLS of wireless communication systems. Concretely, two fundamental questions listed as follows motivate us to study the research of this paper:
1) How to extend the feedback scheme in [27] to the BCMSR-R?

2) For the BC-MSR-R, does a feedback scheme similar to that of [27] still gain advantages over the traditional one used in [20]-[26]?

In this paper, we answer the above two questions by investigating the BC-MSR-R with noiseless feedback, see Figure 1. The main contribution of this paper includes:

1) We propose two inner bounds on the secrecy capacity region of the BC-MSR-R with noiseless feedback, where one is constructed according to the traditional secret key based feedback strategy, and the other is constructed according to a feedback scheme which is an extension of the recently proposed new feedback strategy for the wiretap channel [27].

2) From a Dueck-type example of the BC-MSR-R with noiseless feedback, we show that both the new feedback strategy and the secret key based feedback strategy have their advantages and disadvantages, and both of them even achieve the capacity region of the Dueck-type BC-MSR-R with noiseless feedback and without secrecy constraint for some special cases, i.e., no transmission rate is sacrificed to achieve perfect secrecy.

Here note that the new feedback scheme in [27] combines the Wyner-Ziv (WZ) coding scheme for the source coding with side information [31] with the previous coding scheme in [20], and this WZ scheme is used to generate a cooperative message for the point-to-point channel (one transmitter and one legitimate receiver) model. The key idea of applying the new feedback scheme in [27] to the BC-MSR-R with noiseless feedback is to extend the WZ scheme to a channel model with one transmitter and two receivers, and combine this generalized WZ scheme with the already existing coding scheme in [20] and the secure coding scheme for the BCMSR-R.

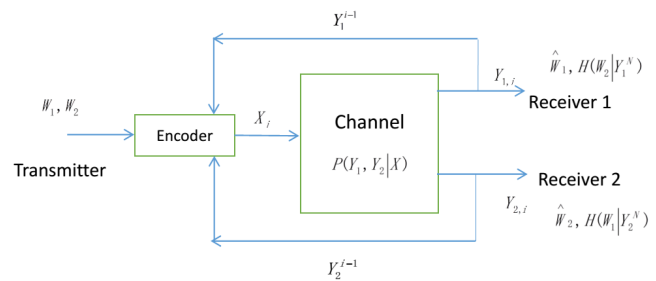

Fig. 1: The broadcast channel with mutual secrecy requirement at the receivers and the noiseless feedback

The outline of this work is organized as follows. Section II gives formal definitions of the system model in Figure 1. Section III introduces the already existing coding schemes of the BC-MSR-R and the distributed source coding with side information, which are key steps in constructing coding schemes for the BC-MSR-R with noiseless feedback. Section IV shows the inner bounds on the secrecy capacity region of the BC-MSR-R with noiseless feedback, and briefly explains the feedback coding schemes achieving these bounds. Section $\mathrm{V}$ proposes an enhanced secret key based feedback scheme for the Dueck-type BC-MSR-R. Section VI provides numerical results to further illustrate the bounds given in the preceding sections. The final conclusion is given in Section VII. 


\section{SySTEM MODEL}

For the rest of this manuscript, the random variables (RVs), values and alphabets are written in uppercase letters, lowercase letters and calligraphic letters, respectively. The random vectors and their values are denoted by a similar convention. For example, $Y_{1}$ represents a RV, and $y_{1}$ represents a value in $\mathcal{Y}_{1}$. Similarly, $Y_{1}^{N}$ represents a random $N$-vector $\left(Y_{1,1}, \ldots, Y_{1, N}\right)$, and $y_{1}^{N}=\left(y_{1,1}, \ldots, y_{1, N}\right)$ represents a vector value in $\mathcal{Y}_{1}^{N}$ (the $N$-th Cartesian power of $\mathcal{Y}_{1}$ ). In addition, for an event $X=x$, its probability is denoted by $P(x)$. In the remainder of this manuscript, the base of the log function is 2 .

The discrete memoryless BC-MSR-R with noiseless feedback has one channel input $x^{N}$, two channel outputs $y_{1}^{N}, y_{2}^{N}$, and the channel transition probability is denoted by

$$
P\left(y_{1}^{N}, y_{2}^{N} \mid x^{N}\right)=\prod_{i=1}^{N} P\left(y_{1, i}, y_{2, i} \mid x_{i}\right),
$$

where $x_{i} \in \mathcal{X}, y_{1, i} \in \mathcal{Y}_{1}$ and $y_{2, i} \in \mathcal{Y}_{2}$.

Let $W_{1}$ and $W_{2}$ be the messages intended to be decoded by Receiver 1 and Receiver 2, respectively. The values of $W_{1}$ and $W_{2}$ belong to the alphabets $\mathcal{W}_{1}=\left\{1,2, \ldots, M_{1}\right\}$ and $\mathcal{W}_{2}=\left\{1,2, \ldots, M_{2}\right\}$, respectively. In addition, $\operatorname{Pr}\left\{W_{1}=\right.$ $i\}=\frac{1}{M_{1}}$ for $i \in \mathcal{W}_{1}$, and $\operatorname{Pr}\left\{W_{2}=j\right\}=\frac{1}{M_{2}}$ for $j \in \mathcal{W}_{2}$. Using noiseless feedback, the transmitter produces the channel input $X_{t}$ at time instant $t$ as a mapping of the messages $W_{1}, W_{2}$ and of the previously received channel outputs $Y_{1}^{t-1}=\left(Y_{1,1}, \ldots, Y_{1, t-1}\right)$ and $Y_{2}^{t-1}=\left(Y_{2,1}, \ldots, Y_{2, t-1}\right)$, i.e.,

$$
X_{t}=f_{t}\left(W_{1}, W_{2}, Y_{1}^{t-1}, Y_{2}^{t-1}\right)
$$

for some stochastic mapping $f_{t}(1 \leq t \leq N)$. Here note that one receiver's feedback is secure from the other receiver.

Receiver $j(j=1,2)$ produces an estimation $\hat{W}_{j}=$ $\psi_{j}\left(Y_{j}^{N}\right)$, where $\psi_{j}$ represents Receiver $j$ 's decoding mapping. Receiver $j$ 's average decoding error probability $P_{e, j}$ equals

$$
P_{e, j}=\frac{1}{M_{j}} \sum_{w_{j} \in \mathcal{W}_{j}} \operatorname{Pr}\left\{\psi_{j}\left(y_{j}^{N}\right) \neq w_{j} \mid w_{j} \text { sent }\right\} .
$$

Receiver 2's equivocation rate of the message $W_{1}$ is formulated as

$$
\Delta_{1}=\frac{1}{N} H\left(W_{1} \mid Y_{2}^{N}\right)
$$

Analogously, Receiver 1's equivocation rate oft the message $W_{2}$ is formulated as

$$
\Delta_{2}=\frac{1}{N} H\left(W_{2} \mid Y_{1}^{N}\right)
$$

Using a similar definition as in [3]-[4], we define a weak achievable secrecy rate pair $\left(R_{1}, R_{2}\right)$ as below. Given two positive numbers $R_{1}$ and $R_{2}$, if for arbitrarily small $\epsilon$, there exist one channel encoder and two channel decoders with parameters $M_{1}, M_{2}, N, \Delta_{1}, \Delta_{2}, P_{e, 1}$ and $P_{e, 2}$ such that

$$
\begin{aligned}
& \frac{\log M_{1}}{N} \geq R_{1}-\epsilon, \frac{\log M_{2}}{N} \geq R_{2}-\epsilon, P_{e, 1} \leq \epsilon, \\
& P_{e, 2} \leq \epsilon, \Delta_{1} \geq R_{1}-\epsilon, \Delta_{2} \geq R_{2}-\epsilon,
\end{aligned}
$$

the pair $\left(R_{1}, R_{2}\right)$ is said to be a weak achievable secrecy rate pair. The secrecy capacity region $\mathcal{C}_{s}^{f}$ consists of all achievable weak secrecy rate pairs, and two inner bounds on $\mathcal{C}_{s}^{f}$ will be given in the remainder of this paper.

\section{PREliminaries}

In this section, first, we review the secure coding scheme of the BC-MSR-R. Next, we introduce an extension of the WZ scheme, which we call the generalized WZ coding scheme [34] used in the problem of distributed source coding with side information. In the next section, the introduced schemes of this section will be used to construct inner bounds on the secrecy capacity region $\mathcal{C}_{s}^{f}$ of the BC-MSR-R with noiseless feedback.

\section{A. Coding scheme for the BC-MSR-R}

For the model of Figure 1 without feedback, a secure coding scheme combining Marton's binning technique [32] for the general broadcast channel and the random binning technique [1] for the PLS is proposed in [3]-[4]. In this subsection, we review this secure coding scheme.

Definitions: The message $W_{j}(j=1,2)$ is conveyed to Receiver $j$, and it is uniformly drawn from the set $\left\{1, \ldots, 2^{N R_{j}}\right\}$. The randomly generated $W_{j}^{\prime}$, which is used for confusing the illegal receiver ${ }^{1}$, is uniformly drawn from the set $\left\{1, \ldots, 2^{N R_{j}^{\prime}}\right\}$, i.e., $\operatorname{Pr}\left\{W_{j}^{\prime}=i\right\}=2^{-N R_{j}^{\prime}}$, where $i \in\left\{1, \ldots, 2^{N R_{j}^{\prime}}\right\}$. Moreover, similar to the coding scheme in Marton's achievable region for the broadcast channel [32], the message $W_{j}^{\prime \prime}$, which enables its codeword $U_{j}^{N}$ to be jointly typical with other codewords, chooses values from the set $\left\{1, \ldots, 2^{N R_{j}^{\prime \prime}}\right\}$.

Code construction: First, randomly generate $2^{N R_{0}}$ i.i.d. $Q^{N}$ with respect to (w.r.t.) $P(q)$, and index them as $q^{N}\left(w_{0}\right)$, where $w_{0} \in\left\{1,2, \ldots, 2^{N R_{0}}\right\}$. Here $Q^{N}$ serves as codeword of a dummy common message $w_{0}$. Then, for each possible value of $q^{N}$, randomly generate $2^{N\left(R_{j}+R_{j}^{\prime}+R_{j}^{\prime \prime}\right)}$ i.i.d. $U_{j}^{N}$ w.r.t. $P\left(u_{j} \mid q\right)$, and index them as $u_{j}^{N}\left(w_{j}, w_{j}^{\prime}, w_{j}^{\prime \prime}\right)$, where $w_{j} \in\left\{1,2, \ldots, 2^{N R_{j}}\right\}, w_{j}^{\prime} \in\left\{1,2, \ldots, 2^{N R_{j}^{\prime}}\right\}$ and $w_{j}^{\prime \prime} \in\left\{1,2, \ldots, 2^{N R_{j}^{\prime \prime}}\right\}$. Finally, for each possible value of $q^{N}, u_{1}^{N}$ and $u_{2}^{N}$, the channel input $x^{N}$ is i.i.d. generated w.r.t. $P\left(x \mid q, u_{1}, u_{2}\right)$. Here note that the construction of $U_{j}^{N}$ combines the random binning scheme [1] ( $w_{j}^{\prime}$ is the dummy message used in [1]) with Marton's binning scheme [32] ( $w_{j}^{\prime \prime}$ is the binning message used in [32]).

Encoding procedure: The transmitter selects $q^{N}\left(w_{0}\right)$, $u_{1}^{N}\left(w_{1}, w_{1}^{\prime}, w_{1}^{\prime \prime}\right)$ and $u_{2}^{N}\left(w_{2}, w_{2}^{\prime}, w_{2}^{\prime \prime}\right)$ to transmit. Here notice that $w_{0}, w_{1}^{\prime}$ and $w_{2}^{\prime}$ are randomly chosen from the sets $\left\{1,2, \ldots, 2^{N R_{0}}\right\},\left\{1,2, \ldots, 2^{N R_{1}^{\prime}}\right\}$ and $\left\{1,2, \ldots, 2^{N R_{2}^{\prime}}\right\}$, respectively, and the indexes $w_{1}^{\prime \prime}$ and $w_{2}^{\prime \prime}$ are chosen by finding a pair of $\left(u_{1}^{N}\left(w_{1}, w_{1}^{\prime}, w_{1}^{\prime \prime}\right), u_{2}^{N}\left(w_{2}, w_{2}^{\prime}, w_{2}^{\prime \prime}\right)\right)$ satisfying the condition that given $q^{N}\left(w_{0}\right),\left(u_{1}^{N}, u_{2}^{N}, q^{N}\left(w_{0}\right)\right)$ are jointly typical.

Decoding procedure: First, Receiver $j(j=1,2)$ chooses a unique $q^{N}$ jointly typical with $y_{j}^{N}$. After decoding $q^{N}$, Receiver $j$ seeks a unique $u_{j}^{N}$ satisfying the condition that $\left(u_{j}^{N}, q^{N}, y_{j}^{N}\right)$ are jointly typical. Once $u_{j}^{N}$ is decoded, Receiver $j$ extracts $w_{j}$ in it.

\footnotetext{
${ }^{1}$ The idea of using random messages to confuse the wiretapper is exactly the same as the random binning scheme used in Wyner's wiretap channel [1], where this randomly produced message is analogous to the randomly chosen bin index used in the random binning scheme.
} 
Based on the above encoding-decoding scheme, an achievable secrecy rate region $\mathcal{C}_{b c-m s r-r}$ for the BC-MSR-R is obtained [3]. Combining the above coding scheme for $\mathcal{C}_{b c-m s r-r}$ with the already existing secret key based feedback scheme [20], it is not difficult to propose a secret key based feedback coding scheme for the BC-MSR-R, which will be shown in the next section.

\section{B. The Generalized Wyner-Ziv Coding Scheme for Distributed Source Coding with Side Information}

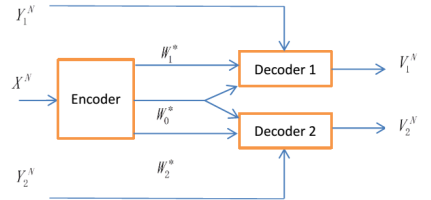

Fig. 2: The distributed source coding with side information

This subsection reviews the generalized WZ scheme for distributed source coding with side information [34], which is the key step in the construction of the feedback scheme for the BC-MSR-R. In Figure 2, a source sequence $X^{N}$ together with two correlated sequences $Y_{1}^{N}$ and $Y_{2}^{N}$ (side information) are i.i.d. produced w.r.t. the joint probability $P\left(x, y_{1}, y_{2}\right)$. Applying an encoding mapping $\phi: \mathcal{X}^{N} \rightarrow$ $\left\{1,2, \ldots, 2^{N R_{0}}\right\} \times\left\{1,2, \ldots, 2^{N R_{1}}\right\} \times\left\{1,2, \ldots, 2^{N R_{2}}\right\}$, a source encoder compresses the source sequence $X^{N}$ into three indexes $W_{0}^{*}, W_{1}^{*}$ and $W_{2}^{*}$, respectively choosing values from the sets $\left\{1,2, \ldots, 2^{N R_{0}}\right\},\left\{1,2, \ldots, 2^{N R_{1}}\right\}$ and $\left\{1,2, \ldots, 2^{N R_{2}}\right\}$. After receiving the indexes $W_{0}^{*}, W_{j}^{*}(j=1,2)$ and the side information $Y_{j}^{N}$, Receiver $j$ produces a reconstruction $\hat{V}_{j}^{N}=\varphi\left(W_{0}^{*}, W_{j}^{*}, Y_{j}^{N}\right)$ via a reconstruction mapping $\varphi_{j}$ : $\left\{1,2, \ldots, 2^{N R_{0}}\right\} \times\left\{1,2, \ldots, 2^{N R_{j}}\right\} \times \mathcal{Y}_{j}^{N} \rightarrow \mathcal{V}_{j}^{N}$. The object of the decoder of Figure 2 is to reconstruct $\hat{V}_{j}^{N}$ which is jointly typical with $X^{N}$ w.r.t. $P\left(v_{j} \mid x\right) \times P(x)$.

A rate triplet $\left(R_{0}, R_{1}, R_{2}\right)$ is called achievable if for any $\epsilon>0$, there exists source encoder and decoders $\left(\phi, \varphi_{1}, \varphi_{2}\right)$ such that

$$
\operatorname{Pr}\left\{\left(X^{N}, \hat{V}_{j}^{N}\right) \notin T_{\epsilon}^{N}\left(P\left(x, v_{j}\right)\right)\right\} \rightarrow 0
$$

while $N \rightarrow \infty$. The following Theorem 1 is a result of the re-treatment of the model in [34], and it provides an achievable region $\mathcal{R}_{\text {inner }}$ consisting of achievable rate triplets $\left(R_{0}, R_{1}, R_{2}\right)$ for this distributed source coding with side information problem.

Theorem 1: (Generalized WZ Theorem): For the distributed source coding with side information, an achievable rate region $\mathcal{R}_{\text {inner }}$ is given by

$$
\begin{aligned}
& \mathcal{R}_{\text {inner }}=\left\{\left(R_{0}, R_{1}, R_{2}\right): R_{0}+R_{1} \geq I\left(X ; V_{0}, V_{1} \mid Y_{1}\right)\right. \\
& R_{0}+R_{2} \geq I\left(X ; V_{0}, V_{2} \mid Y_{2}\right) \\
& R_{0}+R_{1}+R_{2} \geq I\left(X ; V_{1} \mid Y_{1}, V_{0}\right)+I\left(X ; V_{2} \mid Y_{2}, V_{0}\right) \\
& \left.+\max _{j \in\{1,2\}} I\left(X ; V_{0} \mid Y_{j}\right)\right\},
\end{aligned}
$$

where $\left(V_{0}, V_{1}, V_{2}\right) \rightarrow X \rightarrow\left(Y_{1}, Y_{2}\right)$.

Achievability:

The index $w_{0}^{*}$ chooses values from the set $\left\{1,2, \ldots, 2^{N R_{0}}\right\}$, and divide $w_{0}^{*}$ into three sub-indexes $w_{0,0}^{*}, w_{0,1}^{*}$ and $w_{0,2}^{*}$, where each sub-index $w_{0, i}^{*}(i \in\{0,1,2\})$ chooses values from the set $\left\{1,2, \ldots, 2^{N R_{0, i}}\right\}$ and $R_{0,0}+R_{0,1}+R_{0,2}=R_{0}$. The index $w_{1}^{*}$ chooses values from $\left\{1,2, \ldots, 2^{N R_{1}}\right\}$, and divide $w_{1}^{*}$ into two sub-indexes $w_{1,0}^{*}$ and $w_{1,1}^{*}$, where each sub-index $w_{1, j}^{*}(j \in\{0,1\})$ chooses values from $\left\{1,2, \ldots, 2^{N R_{1, j}}\right\}$ and $R_{1,0}+R_{1,1}=R_{1}$. Similarly, the index $w_{2}^{*}$ chooses values from $\left\{1,2, \ldots, 2^{N R_{2}}\right\}$, and divide $w_{2}^{*}$ into two sub-indexes $w_{2,0}^{*}$ and $w_{2,2}^{*}$, where each sub-index $w_{2, l}^{*}(l \in\{0,2\})$ chooses values from $\left\{1,2, \ldots, 2^{N R_{2, l}}\right\}$ and $R_{2,0}+R_{2,2}=R_{2}$. Define $k_{1,0}, k_{2,0}, k_{1}$ and $k_{2}$ as auxiliary indexes respectively taking values in $\left\{1,2, \ldots, 2^{N\left(R_{0}^{\prime}-R_{1,0}\right)}\right\},\left\{1,2, \ldots, 2^{N\left(R_{0}^{\prime}-R_{2,0}\right)}\right\}$, $\left\{1,2, \ldots, 2^{N R_{1}^{\prime}}\right\}$ and $\left\{1,2, \ldots, 2^{N R_{2}^{\prime}}\right\}$.

Code construction: There are two different ways to generate the sequence $v_{0}^{N}$. The first way is to generate $2^{N\left(R_{0,0}+R_{0}^{\prime}\right)}$ i.i.d. sequences $v_{0}^{N}\left(1 ; w_{0,0}^{*}, w_{1,0}^{*}, k_{1,0}\right)$ w.r.t. the probability $P\left(v_{0}\right)$. The second way is to generate $2^{N\left(R_{0,0}+R_{0}^{\prime}\right)}$ i.i.d. sequences $v_{0}^{N}\left(2 ; w_{0,0}^{*}, w_{2,0}^{*}, k_{2,0}\right)$ w.r.t. the probability $P\left(v_{0}\right)$. Here note that $v_{0}^{N}\left(j ; w_{0,0}^{*}, w_{j, 0}^{*}, k_{j, 0}\right)(j \in\{1,2\})$ is intended to be decoded by Receiver $j$. Then, generate $2^{N\left(R_{0,1}+R_{1,1}+R_{1}^{\prime}\right)}$ i.i.d. sequences $v_{1}^{N}\left(w_{0,1}^{*}, w_{1,1}^{*}, k_{1}\right)$ w.r.t. the probability $P\left(v_{1}\right)$, and generate $2^{N\left(R_{0,2}+R_{2,2}+R_{2}^{\prime}\right)}$ i.i.d. sequences $v_{2}^{N}\left(w_{0,2}^{*}, w_{2,2}^{*}, k_{2}\right)$ w.r.t. the probability $P\left(v_{2}\right)$.

Encoding: For a chosen source $x^{N}$, the transmitter seeks a pair of sequences $\left(v_{0}^{N}\left(j ; \tilde{w}_{0,0}^{*}, \tilde{w}_{j, 0}^{*}, \tilde{k}_{j, 0}\right), v_{j}^{N}\left(\tilde{w}_{0, j}^{*}, \tilde{w}_{j, j}^{*}, \tilde{k}_{j}\right)\right)$ such that $\left(x^{N}, v_{0}^{N}\left(j ; \tilde{w}_{0,0}^{*}, \tilde{w}_{j, 0}^{*}, k_{j, 0}\right), v_{j}^{N}\left(\tilde{w}_{0, j}^{*}, \tilde{w}_{j, j}^{*}, \tilde{k}_{j}\right)\right)$ are jointly typical, where $j \in\{1,2\}$. If more than one such pair exist, randomly select one pair. If no such pair exists, an encoding error occurs. According to the covering lemma [33], the encoding error tends to zero if $R_{0}^{\prime}+R_{0,0} \geq$ $I\left(X ; V_{0}\right)$, and $R_{j}^{\prime}+R_{0, j}+R_{j, j} \geq I\left(V_{j} ; X, V_{0}\right)$. Once the sequences $v_{0}^{N}\left(j ; \tilde{w}_{0,0}^{*}, \tilde{w}_{j, 0}^{*}, \tilde{k}_{j, 0}\right)$ and $v_{j}^{N}\left(\tilde{w}_{0, j}^{*}, \tilde{w}_{j, j}^{*}, \tilde{k}_{j}\right)$ are chosen for $j \in\{1,2\}$, the transmitter sends the index $w_{0}^{*}=$ $\left(\tilde{w}_{0,0}^{*}, \tilde{w}_{0,1}^{*}, \tilde{w}_{0,2}^{*}\right)$ to both receivers, sends $w_{1}^{*}=\left(\tilde{w}_{1,0}^{*}, \tilde{w}_{1,1}^{*}\right)$ to Receiver 1 only, and sends $w_{2}^{*}=\left(\tilde{w}_{2,0}^{*}, \tilde{w}_{2,2}^{*}\right)$ to Receiver 2 only.

Decoding: After receiving the indexes $w_{0}^{*}$ and $w_{j}^{*}$ $(j \in\{1,2\})$, Receiver $j$ parses the common index $w_{0}^{*}$ as $\left(\tilde{w}_{0,0}^{*}, \tilde{w}_{0,1}^{*}, \tilde{w}_{0,2}^{*}\right)$, and its private index $w_{j}^{*}$ as $\left(\tilde{w}_{j, 0}^{*}, \tilde{w}_{j, j}^{*}\right)$. Then given the side information $y_{j}^{N}$ and $\tilde{w}_{0,0}^{*}$, $\tilde{w}_{0,1}^{*}, \quad \tilde{w}_{0,2}^{*}, \quad \tilde{w}_{j, 0}^{*}, \quad \tilde{w}_{j, j}^{*}$, Receiver $j$ seeks a unique pair of $\left(v_{0}^{N}\left(j ; \tilde{w}_{0,0}^{*}, \tilde{w}_{j, 0}^{*}, \hat{k}_{j, 0}\right), v_{j}^{N}\left(\tilde{w}_{0, j}^{*}, \tilde{w}_{j, j}^{*}, \hat{k}_{j}\right)\right)$ satisfying the condition that $\left(v_{0}^{N}\left(j ; \tilde{w}_{0,0}^{*}, \tilde{w}_{j, 0}^{*}, \hat{k}_{j, 0}\right), v_{j}^{N}\left(\tilde{w}_{0, j}^{*}, \tilde{w}_{j, j}^{*}, \hat{k}_{j}\right), y_{j}^{N}\right)$ are jointly typical. If no or more than one pair exist, an decoding error occurs. According to the packing lemma [33], Receiver $j$ 's decoding error tends to zero if $R_{j}^{\prime} \leq I\left(V_{j} ; V_{0}, Y_{j}\right)$, and $R_{0}^{\prime}-R_{j, 0}+R_{j}^{\prime} \leq I\left(V_{0} ; Y_{j}\right)+$ $I\left(V_{j} ; V_{0}, Y_{j}\right)$. Once Receiver $j$ finds such unique pair of $\left(v_{0}^{N}\left(j ; \tilde{w}_{0,0}^{*}, \tilde{w}_{j, 0}^{*}, \hat{k}_{j, 0}\right), v_{j}^{N}\left(\tilde{w}_{0, j}^{*}, \tilde{w}_{j, j}^{*}, \hat{k}_{j}\right)\right)$, he produces the re-construction sequence $\hat{V}_{j}^{N}=v_{j}^{N}\left(\tilde{w}_{0, j}^{*}, \tilde{w}_{j, j}^{*}, \hat{k}_{j}\right)$.

Using the fact that $R_{0,0}+R_{0,1}+R_{0,2}=R_{0}, R_{1,0}+R_{1,1}=$ $R_{1}, R_{2,0}+R_{2,2}=R_{2}$, and applying Fourier-Motzkin elimination to remove $R_{0}^{\prime}, R_{1}^{\prime}$ and $R_{2}^{\prime}$ from the above constraint inequalities, Theorem 1 is obtained. Here note that the generalized WZ scheme described above indicates that in a broadcast channel, each receiver's channel output can be viewed as side information helping him to decode an estimation of the 
channel input, and this estimation of the channel input further helps the receiver to improve his/her decoding performance. Motivated by this scheme, in the next section, a new feedback strategy for the BC-MSR-R is proposed, which combines the already existing secret key based feedback scheme [20] and the generalized WZ scheme with the previous secure coding scheme for the BC-MSR-R described in Subsection III-A.

\section{Main Results of this PAPER}

In this section, we first propose a new inner bound $\mathcal{C}_{s}^{f-i n-2}$ on $\mathcal{C}_{s}^{f}$. The feedback channel outputs $Y_{1}^{i-1}$ and $Y_{2}^{i-1}$ are not only used to generate secret keys protecting part of the messages, but also used to produce cooperative messages represented by $V_{0}, V_{1}$ and $V_{2}$ helping the receivers to improve their decoding performance. The inner bound $\mathcal{C}_{s}^{f-i n-2}$ is provided in the following Theorem 2 .

Theorem 2: $\mathcal{C}_{s}^{f-i n-2} \subseteq \mathcal{C}_{s}^{f}$, where

$$
\begin{aligned}
& \mathcal{C}_{s}^{f-i n-2}=\left\{\left(R_{1}, R_{2}\right): R_{1} \leq \min \left\{\left[I\left(U_{1} ; Y_{1}, V_{1} \mid Q\right)\right.\right.\right. \\
& \left.-I\left(U_{1} ; U_{2} \mid Q\right)-I\left(U_{1} ; Y_{2}, V_{2} \mid Q, U_{2}\right)\right]^{+} \\
& \left.+H\left(Y_{1} \mid Q, U_{1}, U_{2}, Y_{2}, V_{2}\right), I\left(U_{1} ; Y_{1}, V_{1} \mid Q\right)\right\}, \\
& R_{1} \leq I\left(Q, U_{1} ; V_{1}, Y_{1}\right)+I\left(U_{2} ; V_{1}, Y_{1} \mid Q, U_{1}\right) \\
& -I\left(U_{1} ; U_{2} \mid Q\right)-I\left(V_{1} ; \tilde{X} \mid Y_{1}, V_{0}\right) \\
& -I\left(V_{2} ; \tilde{X} \mid Y_{2}, V_{0}\right)-\max _{j \in\{1,2\}} I\left(V_{0} ; \tilde{X} \mid Y_{j}\right), \\
& R_{2} \leq \min \left\{\left[I\left(U_{2} ; Y_{2}, V_{2} \mid Q\right)-I\left(U_{1} ; U_{2} \mid Q\right)\right.\right. \\
& \left.-I\left(U_{2} ; Y_{1}, V_{1} \mid Q, U_{1}\right)\right]^{+}+H\left(Y_{2} \mid Q, U_{1}, U_{2}, Y_{1}, V_{1}\right), \\
& \left.I\left(U_{2} ; Y_{2}, V_{2} \mid Q\right)\right\}, \\
& R_{2} \leq I\left(Q, U_{2} ; V_{2}, Y_{2}\right)+I\left(U_{1} ; V_{2}, Y_{2} \mid Q, U_{2}\right) \\
& -I\left(U_{1} ; U_{2} \mid Q\right)-I\left(V_{1} ; \tilde{X} \mid Y_{1}, V_{0}\right) \\
& -I\left(V_{2} ; \tilde{X} \mid Y_{2}, V_{0}\right)-\max _{j \in\{1,2\}} I\left(V_{0} ; \tilde{X} \mid Y_{j}\right), \\
& R_{1}+R_{2} \leq I\left(U_{1} ; V_{1}, Y_{1} \mid Q\right)+I\left(U_{2} ; V_{2}, Y_{2} \mid Q\right) \\
& +\min _{j \in\{1,2\}} I\left(Q ; V_{j}, Y_{j}\right)-I\left(U_{1} ; U_{2} \mid Q\right)-I\left(V_{1} ; \tilde{X} \mid Y_{1}, V_{0}\right) \\
& -I\left(V_{2} ; \tilde{X} \mid Y_{2}, V_{0}\right)-\max _{j \in\{1,2\}} I\left(V_{0} ; \tilde{X} \mid Y_{j}\right), \\
& R_{1}+R_{2} \leq I\left(U_{1} ; Y_{1}, V_{1} \mid Q\right)+I\left(U_{2} ; Y_{2}, V_{2} \mid Q\right) \\
& -I\left(U_{1} ; U_{2} \mid Q\right), \\
& R_{1}+R_{2} \leq I\left(Q, U_{1} ; V_{1}, Y_{1}\right)+I\left(Q, U_{2} ; V_{2}, Y_{2}\right) \\
& -I\left(U_{1} ; U_{2} \mid Q\right)-I\left(V_{0}, V_{1} ; \tilde{X} \mid Y_{1}\right)-I\left(V_{0}, V_{2} ; \tilde{X} \mid Y_{2}\right), \\
& R_{1}+2 R_{2} \leq I\left(U_{1} ; V_{1}, Y_{1} \mid Q\right)+2 I\left(Q, U_{2} ; V_{2}, Y_{2}\right) \\
& -2 I\left(U_{1} ; U_{2} \mid Q\right)-I\left(U_{2} ; V_{1}, Y_{1} \mid Q, U_{1}\right) \\
& +H\left(Y_{2} \mid Q, U_{1}, U_{2}, Y_{1}, V_{1}\right) \\
& -I\left(V_{0}, V_{1} ; \tilde{X} \mid Y_{1}\right)-I\left(V_{0}, V_{2} ; \tilde{X} \mid Y_{2}\right), \\
& 2 R_{1}+R_{2} \leq I\left(U_{2} ; V_{2}, Y_{2} \mid Q\right)+2 I\left(Q, U_{1} ; V_{1}, Y_{1}\right) \\
& -2 I\left(U_{1} ; U_{2} \mid Q\right)-I\left(U_{1} ; V_{2}, Y_{2} \mid Q, U_{2}\right) \\
& +H\left(Y_{1} \mid Q, U_{1}, U_{2}, Y_{2}, V_{2}\right)-I\left(V_{0}, V_{1} ; \tilde{X} \mid Y_{1}\right) \\
& \left.-I\left(V_{0}, V_{2} ; \tilde{X} \mid Y_{2}\right)\right\}, \\
& \\
& \left.+f_{1}\right)
\end{aligned}
$$

$[x]^{+}=x$ for $x \geq 0,[x]^{+}=0$ for $x<0, \tilde{X}=$ $\left(Q, U_{1}, U_{2}, Y_{1}, Y_{2}\right)$ and the joint distribution is denoted by

$$
\begin{aligned}
& P\left(v_{0}, v_{1}, v_{2} \mid q, u_{1}, u_{2}, y_{1}, y_{2}\right) P\left(y_{1}, y_{2} \mid x\right) . \\
& P\left(x \mid u_{1}, u_{2}, q\right) P\left(u_{1}, u_{2}, q\right) .
\end{aligned}
$$

\section{Proof sketch:}

The coding scheme achieving the inner bound $\mathcal{C}_{s}^{f-i n-2}$ combines the already existing secret key based feedback scheme [20] and the previous coding scheme for the BC-MSR$\mathrm{R}$ with the generalized WZ scheme described in Section III, and it can be briefly illustrated as follows.

Encoding: The transmission is through $n$ blocks, and each block has length $N$. First, similar to the secret key based feedback scheme [20], in each block, split the transmitted message $w_{i}(i \in\{1,2\})$ into two parts, i.e., $w_{i}=\left(w_{i, 1}, w_{i, 2}\right)$. The sub-message $w_{i, 1}$ is encoded exactly the same as that in the coding scheme for BC-MSR-R (see Section III), and $w_{i, 2}$ is encrypted by a key produced by the feedback channel output $y_{i}^{N}$ of the previous block. Then, compress the encoded sequences $u_{1}^{N}, u_{2}^{N}, q^{N}$ and the feedback channel outputs $y_{1}^{N}$ and $y_{2}^{N}$ from the previous block into three indexes $w_{0}^{*}, w_{1}^{*}$ and $w_{2}^{*}$. Similar to the generalized $\mathrm{WZ}$ scheme introduced in Section III, we use the indexes $w_{0}^{*}, w_{1}^{*}$ and $w_{2}^{*}$ to generate $v_{1}^{N}$, $v_{2}^{N}$ and $v_{0}^{N}$, where $v_{i}^{N}(i \in\{1,2\})$ is Receiver $i$ 's estimation of the channel input, and $v_{0}^{N}$ is an auxiliary sequence helping Receiver $i$ to decode $v_{i}^{N}$. Finally, the sequence $u_{i}^{N}(i \in\{1,2\})$ for each block is chosen according to the current block's $w_{i, 1}$, similar auxiliary messages $w_{i}^{\prime}, w_{i}^{\prime \prime}$ shown in the coding scheme for BC-MSR-R, the encrypted $w_{i, 2}$ and the previous block's compressed index $w_{i}^{*}$. Moreover, the sequence $q^{N}$ is chosen according to the current block's randomly chosen "common message" $w_{0}$ (see the coding scheme for BC-MSR$\mathrm{R}$ in Section III) and the previous block's compressed index $w_{0}^{*}$. Here note that for the last block, we do not transmit the real message $w_{i}(i \in\{1,2\})$ to Receiver $i$, i.e., we transmit a constant in block $n$.

Decoding: The decoding for Receiver $i$ ( $i \in\{1,2\})$ begins from the last block. In block $n$, using a similar decoding scheme of the non-feedback coding scheme for BC-MSR$\mathrm{R}$, Receiver $i$ decodes $u_{i}^{N}$ and $q^{N}$ for block $n$. Then he extracts the block $n-1$ 's compressed indexes $w_{i}^{*}$ and $w_{0}^{*}$ from the decoded $u_{i}^{N}$ and $q^{N}$ of block $n$, respectively. Next, similar to the generalized WZ scheme, Receiver $i$ views the received signal $y_{i}^{N}$ of block $n-1$ as side information. Given block $n-1$ 's $w_{i}^{*}, w_{0}^{*}$ and $y_{i}^{N}$, Receiver $i$ seeks a unique pair of $\left(v_{i}^{N}, v_{0}^{N}\right)$ in block $n-1$ satisfying the condition that $\left(v_{i}^{N}, v_{0}^{N}, y_{i}^{N}\right)$ are jointly typical. Once $v_{i}^{N}$ of block $n-1$ is decoded, Receiver $i$ decodes $q^{N}$ and $u_{i}^{N}$ for block $n-1$ by finding a unique pair $\left(q^{N}, u_{i}^{N}\right)$ satisfying the condition that $\left(u_{i}^{N}, q^{N}, y_{i}^{N}, v_{i}^{N}\right)$ are jointly typical. Once Receiver $i$ decodes $u_{i}^{N}$ and $q^{N}$ for block $n-1$, he obtains the transmitted message $w_{i}$ for block $n-1$ and extracts the block $n-2$ 's compressed indexes $w_{i}^{*}$ and $w_{0}^{*}$. Repeating the above decoding procedure, Receiver $i$ obtains all the messages. Details about the proof are in Appendix A.

The following inner bound $\mathcal{C}_{s}^{f-i n-1}$ in Corollary 1 can be directly obtained from Theorem 2 by letting $V_{0}, V_{1}$ and $V_{2}$ (the estimation of the channel input) of $\mathcal{C}_{s}^{f-i n-2}$ be constants (hence degrading the WZ scheme), and this bound can be viewed as a secret key based inner bound (application of the secret key based feedback strategy [20] to the BC-MSR-R with noiseless feedback) on $\mathcal{C}_{s}^{f}$.

Corollary 1: $\mathcal{C}_{s}^{f-i n-1} \subseteq \mathcal{C}_{s}^{f}$, where

$$
\begin{aligned}
& \mathcal{C}_{s}^{f-i n-1}=\left\{\left(R_{1}, R_{2}\right): R_{1} \leq \min \left\{\left[I\left(U_{1} ; Y_{1} \mid Q\right)\right.\right.\right. \\
& \left.-I\left(U_{1} ; U_{2} \mid Q\right)-I\left(U_{1} ; Y_{2} \mid Q, U_{2}\right)\right]^{+}
\end{aligned}
$$




$$
\begin{aligned}
& \left.+H\left(Y_{1} \mid Q, U_{1}, U_{2}, Y_{2}\right), I\left(U_{1} ; Y_{1} \mid Q\right)\right\}, \\
& R_{1} \leq I\left(Q, U_{1} ; Y_{1}\right)+I\left(U_{2} ; Y_{1} \mid Q, U_{1}\right)-I\left(U_{1} ; U_{2} \mid Q\right), \\
& R_{2} \leq \min \left\{\left[I\left(U_{2} ; Y_{2} \mid Q\right)-I\left(U_{1} ; U_{2} \mid Q\right)-\right.\right. \\
& \left.\left.I\left(U_{2} ; Y_{1} \mid Q, U_{1}\right)\right]^{+}+H\left(Y_{2} \mid Q, U_{1}, U_{2}, Y_{1}\right), I\left(U_{2} ; Y_{2} \mid Q\right)\right\} \\
& R_{2} \leq I\left(Q, U_{2} ; Y_{2}\right)+I\left(U_{1} ; Y_{2} \mid Q, U_{2}\right)-I\left(U_{1} ; U_{2} \mid Q\right), \\
& \left.R_{1}+R_{2} \leq I\left(U_{1} ; Y_{1} \mid Q\right)+I\left(U_{2} ; Y_{2} \mid Q\right)-I\left(U_{1} ; U_{2} \mid Q\right)\right\},
\end{aligned}
$$

and the joint distribution is denoted by

$$
P\left(y_{1}, y_{2} \mid x\right) P\left(x \mid u_{1}, u_{2}\right) P\left(u_{1}, u_{2} \mid q\right) P(q) .
$$

Remark 1: In $\mathcal{C}_{s}^{f-i n-2}$, the feedback $Y_{1}^{i-1}$ and $Y_{2}^{i-1}$ are not only used to generate secret keys, but also used to produce cooperative messages represented by $V_{0}, V_{1}$ and $V_{2}$. If $V_{0}, V_{1}$ and $V_{2}$ are defined to be constants, then there is no cooperative message generated from the feedback, which implies that the feedback is only used to generate secret keys protecting part of the transmitted messages, and this feedback scheme can be viewed as an extension of the secret key based feedback scheme for the wiretap channel [20] to the BC-MSR-R. Hence substituting $V_{0}=V_{1}=V_{2}=$ const into $\mathcal{C}_{s}^{f-i n-2}$, and along the lines of the proof of $\mathcal{C}_{s}^{f-i n-2}$, we obtain the secret key based inner bound $\mathcal{C}_{s}^{f-i n-1}$.

For a special case of BC-MSR-R, where the broadcast channel consists of a broadcast sub-channel and private subchannels, an enhanced secret key based feedback scheme is proposed (see Section V), and it is shown to be better than the traditional secret key based feedback scheme given in Corollary 1.

\section{An Enhanced Secret Key Based FeEdback SCHEME FOR A SPECIAL CASE OF BC-MSR-R}

In this section, we propose an enhanced secret key based feedback scheme for a special case of BC-MSR-R, which we call the Dueck-type BC-MSR-R (see Figure 3). For the model of Figure 3, the channel inputs and outputs are given by

$$
\begin{aligned}
& X=\left(X_{0}, X_{1}, X_{2}\right), Y_{1}=\left(Y_{10}, Y_{11}\right), Y_{2}=\left(Y_{20}, Y_{21}\right), \\
& Y_{10}=Y_{20}=X_{0} \oplus Z_{0}, Y_{11}=X_{1} \oplus Z_{1}, Y_{21}=X_{2} \oplus Z_{2},
\end{aligned}
$$

where $X_{0}, X_{1}, X_{2}, Z_{0}, Z_{1}, Z_{2}$ take values in $\{0,1\}$, and the channel noises are independent of the channel inputs.

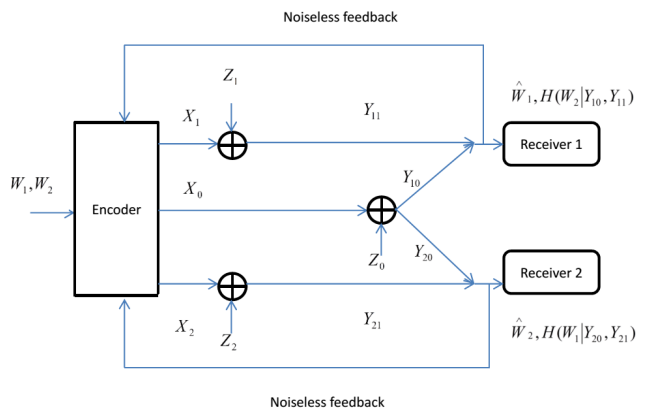

Fig. 3: The Dueck-type BC-MSR-R with noiseless feedback

The secrecy capacity region of this Dueck-type BC-MSR-R with noiseless feedback is denoted by $\mathcal{C}_{s}^{f}$. In this section, we propose an enhanced secret key based inner bound $\mathcal{C}_{s}^{f-i n-1 *}$ on $\mathcal{C}_{s}^{f}$, and in the next section, we will show that this enhanced secret key based inner bound may be larger than the traditional one, and it may be even larger than the new inner bound proposed in Theorem 2. The details about the construction of this enhanced secret key based inner bound are given below.

Case 1: The private channel for receiver 1 transmits part of the secret message $W_{1}$, and the perfect secrecy is guaranteed by Wyner's random binning scheme [1]. Using receiver 1's feedback $Y_{11}$ (here note that $Y_{10}$ is not used because the eavesdropper also knows it) to generate secret key and using this key to encrypt another part of $W_{1}$, the common channel is used to transmit the encrypted part of $W_{1}$, and the perfect secrecy is guaranteed by the secret key. In addition, the private channel for receiver 2 combining with receiver 2 's feedback $Y_{21}$ (here note that $Y_{20}$ is not used because the eavesdropper, i.e., receiver 1 , also knows it) are used to transmit the secret message $W_{2}$, and the perfect secrecy is guaranteed by Ahlswede and Cai's scheme [20]. For case 1 , this scheme can be illustrated by the following Figure 4. Here note that the described scheme in case 1 can achieve the maximum achievable secrecy rate $R_{1}$ due to the reason that the common channel is fully used by receiver 1 , and receiver 2 is not allowed to use it.

Case 2: The private channel for receiver 2 transmits part of the secret message $W_{2}$. Using receiver 2's feedback $Y_{21}$ to generate secret key and using this key to encrypt another part of $W_{2}$, the common channel is used to transmit the encrypted part of $W_{2}$. In addition, the private channel for receiver 1 combining with receiver 1's feedback $Y_{11}$ are used to transmit $W_{1}$. Here note that the described scheme in case 2 can achieve the maximum achievable secrecy rate $R_{2}$ due to the reason that the common channel is fully used by receiver 2 , and receiver 1 is not allowed to use it.

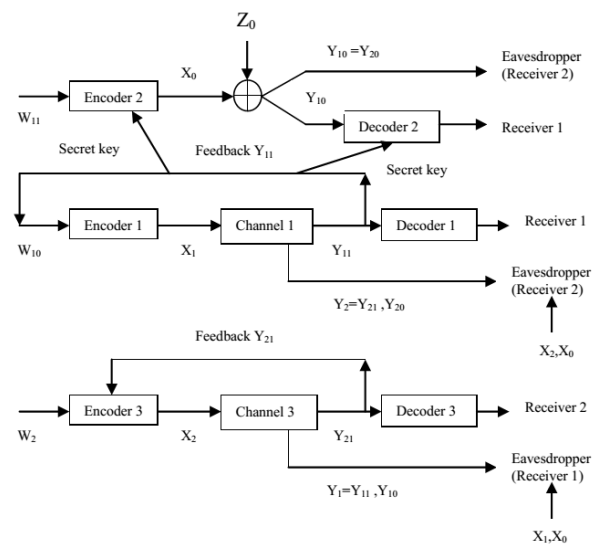

Fig. 4: An enhanced secret key based feedback scheme that achieves the maximum achievable secrecy rate $R_{1}$

For case 1 , the private channel for receiver 1 with eavesdropper (receiver 2 ) can be modeled as a wiretap channel with input $X_{1}$, and outputs $Y_{11}$ and $Y_{2}$ (see Figure 4). Moreover, note that the eavesdropper (receiver 2) can also decode $X_{0}$ and $X_{2}$, using Wyner's random binning scheme [1], the perfect secrecy of the private channel for receiver 1 is guaranteed if the rate is no larger than $\left[I\left(X_{1} ; Y_{11}\right)-I\left(X_{1} ; Y_{2} \mid X_{2}, X_{0}\right)\right]^{+}$, where the function [.] $]^{+}$guarantees that if $I\left(X_{1} ; Y_{11}\right)<$ $I\left(X_{1} ; Y_{2} \mid X_{2}, X_{0}\right)$, there is no positive secrecy rate. Since the feedback of the private channel for receiver 1 is used to 
encrypt the message transmitted over the common channel, the achievable secrecy rate of the common channel depends on the rate of the secret key. First, note that the rate of the key cannot exceed the eavesdropper's equivocation about the feedback, i.e., $H\left(Y_{11} \mid Y_{2}, X_{2}, X_{0}\right)$. Next, note that the rate of the key cannot exceed the capacity of the common channel, i.e., $I\left(X_{0} ; Y_{10}\right)$. Combining the above rates of the private channel for receiver 1 and the common channel, we have

$$
\begin{aligned}
& R_{1} \leq\left[I\left(X_{1} ; Y_{11}\right)-I\left(X_{1} ; Y_{2} \mid X_{2}, X_{0}\right)\right]^{+} \\
& +\min \left\{H\left(Y_{11} \mid Y_{2}, X_{2}, X_{0}\right), I\left(X_{0} ; Y_{10}\right)\right\} .
\end{aligned}
$$

On the other hand, the private channel for receiver 2 with feedback and eavesdropper (receiver 1) can be modeled as the wiretap channel with feedback (see Figure 3). Using Ahlswede and Cai's scheme [20], the perfect secrecy of the private channel for receiver 2 is guaranteed if the rate $R_{2}$ is no larger than

$$
\begin{aligned}
& R_{2} \leq \min \left\{\left[I\left(X_{2} ; Y_{21}\right)-I\left(X_{2} ; Y_{1} \mid X_{1}, X_{0}\right)\right]^{+}\right. \\
& \left.+H\left(Y_{21} \mid Y_{1}, X_{2}, X_{1}, X_{0}\right), I\left(X_{2} ; Y_{21}\right)\right\} .
\end{aligned}
$$

Calculating (5.2) and (5.3), we obtain

$$
\begin{aligned}
& \mathcal{C}_{1}=\left\{\left(R_{1}, R_{2}\right): R_{1} \leq 2-H\left(Z_{0}\right)-H\left(Z_{1}\right),\right. \\
& \left.R_{2} \leq 1-H\left(Z_{2}\right),\right\}
\end{aligned}
$$

where $\mathcal{C}_{1}$ is achieved when $X_{0}, X_{1}, X_{2}$ are independent of one another, and $P\left(X_{0}=0\right)=P\left(X_{1}=0\right)=P\left(X_{2}=0\right)=$ $\frac{1}{2}$. Analogously, for case 2 , we obtain

$$
\begin{aligned}
& \mathcal{C}_{2}=\left\{\left(R_{1}, R_{2}\right): R_{1} \leq 1-H\left(Z_{1}\right),\right. \\
& \left.R_{2} \leq 2-H\left(Z_{0}\right)-H\left(Z_{2}\right),\right\}
\end{aligned}
$$

where $\mathcal{C}_{2}$ is achieved when $X_{0}, X_{1}, X_{2}$ are independent of one another, and $P\left(X_{0}=0\right)=P\left(X_{1}=0\right)=P\left(X_{2}=0\right)=$ $\frac{1}{2}$.

Note that the achievable secrecy rate region $\mathcal{C}_{s}^{f-i n-1 *}$ of the enhanced secret key based feedback scheme is the convex hull of $\mathcal{C}_{1} \cup \mathcal{C}_{2}$, and hence we have

$$
\begin{aligned}
& \mathcal{C}_{s}^{f-i n-1 *}=\left\{\left(R_{1}, R_{2}\right): R_{1} \leq 2-H\left(Z_{0}\right)-H\left(Z_{1}\right),\right. \\
& R_{2} \leq 2-H\left(Z_{0}\right)-H\left(Z_{2}\right), \\
& \left.R_{1}+R_{2} \leq 3-H\left(Z_{0}\right)-H\left(Z_{1}\right)-H\left(Z_{2}\right) .\right\}
\end{aligned}
$$

For comparison, in the remainder of this section, we also calculate the inner bound $\mathcal{C}_{s, n f b}^{\text {in }}$ on the secrecy capacity region of the Dueck-type BC-MSR-R without feedback, the traditional secret key based inner bound $\mathcal{C}_{s}^{f-i n-1}$ on the secrecy capacity region $\mathcal{C}_{s}^{f}$ of the Dueck-type BC-MSR-R with noiseless feedback, the new inner bound $\mathcal{C}_{s}^{f-i n-2}$ on $\mathcal{C}_{s}^{f}$, and a simple cut-set outer bound $\mathcal{C}_{s}^{f-o u t}$ on $\mathcal{C}_{s}^{f}$. First, we calculate the inner bound $\mathcal{C}_{s, n f b}^{\text {in }}$ on the secrecy capacity region of the Dueck-type BC-MSR-R without feedback. Substituting (5.1) into the achievable secrecy rate region $\mathcal{C}_{b c-m s r-r}$ for the $\mathrm{BC}$ MSR-R [3], $\mathcal{C}_{s, n f b}^{i n}$ is given by

$$
\begin{aligned}
& \mathcal{C}_{s, n f b}^{i n}=\left\{\left(R_{1}, R_{2}\right): R_{1} \leq 1-H\left(Z_{1} \mid Z_{0}\right),\right. \\
& \left.R_{2} \leq 1-H\left(Z_{2} \mid Z_{0}\right)\right\},
\end{aligned}
$$

where (5.7) is achieved when $Q=X_{0}, U_{1}=X_{1}, U_{2}=X_{2}$, the RVs $X_{0}, X_{1}, X_{2}$ are independent of one another, and $P\left(X_{0}=0\right)=P\left(X_{1}=0\right)=P\left(X_{2}=0\right)=\frac{1}{2}$.

Second, we calculate the traditional secret key based inner bound $\mathcal{C}_{s}^{f-i n-1}$ on the secrecy capacity region $\mathcal{C}_{s}^{f}$ of the Dueck-type BC-MSR-R with noiseless feedback. Using a similar argument in [35], and substituting (5.1) into Corollary 1 , the inner bound $\mathcal{C}_{s}^{f-i n-1}$ is given by

$$
\begin{aligned}
& \mathcal{C}_{s}^{f-i n-1}=\left\{\left(R_{1}, R_{2}\right): 0 \leq R_{1} \leq 1-H\left(Z_{1} \mid Z_{0}\right),\right. \\
& \left.0 \leq R_{2} \leq 1-H\left(Z_{2} \mid Z_{0}\right)\right\},
\end{aligned}
$$

where (5.8) is achieved when $Q=X_{0}, U_{1}=X_{1}, U_{2}=X_{2}$, the RVs $X_{0}, X_{1}, X_{2}$ are independent of one another, and $P\left(X_{0}=0\right)=P\left(X_{1}=0\right)=P\left(X_{2}=0\right)=\frac{1}{2}$. Comparing the above $\mathcal{C}_{s}^{f-i n-1}$ with $\mathcal{C}_{s, n f b}^{i n}$, we find that the two regions are the same. The intuition behind this fact is that the region $\mathcal{C}_{s}^{f-i n-1}$ is constructed by using the common channel to transmit the auxiliary common message $Q$, and using the private channel for receiver 1 (2) to transmit the secret message $U_{1}\left(U_{2}\right)$ for receiver $1(2)$. Now since the inner bound on the secrecy capacity region of the non-feedback model is the same as $\mathcal{C}_{s}^{f-i n-1}$, which implies that Wyner's random binning scheme achieves the capacity $1-H\left(Z_{1} \mid Z_{0}\right)\left(1-H\left(Z_{2} \mid Z_{0}\right)\right)$ of the private channel for receiver 1 (receiver 2 ) without the eavesdropper, and this leads to the fact that feedback is useless because the secrecy capacity of each private channel with feedback can not exceed the capacity of the private channel without the eavesdropper. The above statement indicates that the proposed secret key based feedback scheme does not perform well in the Dueck-type BC-MSR-R with noiseless feedback.

Third, we show the new inner bound $\mathcal{C}_{s}^{f-i n-2}$ on the secrecy capacity region of the Dueck-type BC-MSR-R with noiseless feedback. Substituting $Q=X_{0}, U_{1}=X_{1}, U_{2}=X_{2}, V_{1}=$ $\left(X_{0}, X_{1}\right), V_{2}=\left(X_{0}, X_{2}\right),(5.1), V_{0}=\left(Z_{0}, Z_{1}\right)$ or $V_{0}=$ $\left(Z_{0}, Z_{2}\right)$ into Theorem 2 , and maximizing the joint probability $P\left(x_{0}, x_{1}, x_{2}\right), \mathcal{C}_{s}^{f-i n-2}$ is given by

$$
\begin{aligned}
& \mathcal{C}_{s}^{f-i n-2}=\left\{\left(R_{1}, R_{2}\right): R_{1} \leq 1, R_{2} \leq 1\right. \\
& \left.R_{1} \leq\left[2-H\left(Z_{0}, Z_{1}, Z_{2}\right)\right]^{+}, R_{2} \leq\left[2-H\left(Z_{0}, Z_{1}, Z_{2}\right)\right]^{+}\right\} .
\end{aligned}
$$

Note that (5.9) is achieved when $X_{0}, X_{1}, X_{2}$ are independent of one another, and $P\left(X_{0}=0\right)=P\left(X_{1}=0\right)=P\left(X_{2}=\right.$ $0)=\frac{1}{2}$.

Finally, we show a simple cut-set outer bound $\mathcal{C}_{s}^{f-o u t}$ on the secrecy capacity region of the Dueck-type BC-MSR-R with noiseless feedback. Since only $X_{0}$ and $X_{1}$ are transmitted to receiver 1 , the transmission rate $R_{1}$ of the message $W_{1}$ is upper bounded by $I\left(X_{0}, X_{1} ; Y_{1}\right)$. Analogously, the transmission rate $R_{2}$ is upper bounded by $I\left(X_{0}, X_{2} ; Y_{2}\right)$. For all receivers, the sum rate $R_{1}+R_{2}$ is upper bounded by $I\left(X_{0}, X_{1}, X_{2} ; Y_{1}, Y_{2}\right)$. By calculating, it is not difficult to show that the cut-set outer bound $\mathcal{C}_{s}^{f-\text { out }}$ on the secrecy capacity region of the Dueck-type BC-MSR-R with noiseless feedback is given by

$$
\begin{aligned}
& \mathcal{C}_{s}^{f-\text { out }}=\left\{\left(R_{1}, R_{2}\right): R_{1} \leq 2-H\left(Z_{0}, Z_{1}\right),\right. \\
& \left.R_{2} \leq 2-H\left(Z_{0}, Z_{2}\right), R_{1}+R_{2} \leq 3-H\left(Z_{0}, Z_{1}, Z_{2}\right)\right\},
\end{aligned}
$$

where (5.10) is achieved when $X_{0}, X_{1}, X_{2}$ are independent of one another, and $P\left(X_{0}=0\right)=P\left(X_{1}=0\right)=P\left(X_{2}=\right.$ $0)=\frac{1}{2}$. Here note that the outer bound $\mathcal{C}_{s}^{f-o u t}$ also serves as an outer bound for the broadcast channel model without secrecy constraint. In the next section, we will show that both the enhanced secret key based inner bound $\mathcal{C}_{s}^{f-i n-1 *}$ and the new inner bound $\mathcal{C}_{s}^{f-i n-2}$ achieve this outer bound for some 
special cases, which implies that the feedback helps to push the secure capacity up to the non-secure one.

\section{NumERICAL RESUlTS}

In order to compare the bounds proposed in Section $\mathrm{V}$, we further define the channel noises $Z_{0}, Z_{1}$ and $Z_{2}$ of the Duecktype example in the following three cases:

- Case 1: The channel noises $Z_{0}, Z_{1}$ and $Z_{2}$ are independent of one another.

- Case 2: Given $Z_{1}, Z_{2}$ is independent of $Z_{0}$, i.e., there exists a Markov chain $Z_{0} \rightarrow Z_{1} \rightarrow Z_{2}$.

- Case 3: Given $Z_{0}, Z_{1}$ is independent of $Z_{2}$, i.e., there exists a Markov chain $Z_{1} \rightarrow Z_{0} \rightarrow Z_{2}$.

In the remainder of this section, we show the numerical results on the above three cases of this Dueck-type example, see the followings.

\section{A. A special case of Dueck-type example with independent $Z_{0}$,} $Z_{1}$ and $Z_{2}$

For this case, define $Z_{0} \sim \operatorname{Bern}(p), Z_{1} \sim \operatorname{Bern}(q)$ and $Z_{2} \sim \operatorname{Bern}(r)$. Then, the traditional secret key based inner bound $\mathcal{C}_{s}^{f-i n-1}$, the enhanced secret key based inner bound $\mathcal{C}_{s}^{f-i n-1 *}$, the new inner bound $\mathcal{C}_{s}^{f-i n-2}$, the outer bound $\mathcal{C}_{s}^{f-o u t}$ and the achievable secrecy rate region $\mathcal{C}_{s, n f b}^{\text {in }}$ for the non-feedback model are given by

$$
\begin{aligned}
& \mathcal{C}_{s}^{f-i n-1}=\mathcal{C}_{s, n f b}^{\text {in }}=\left\{\left(R_{1}, R_{2}\right): 0 \leq R_{1} \leq 1-h(q)\right. \\
& \left.0 \leq R_{2} \leq 1-h(r)\right\} \\
& \mathcal{C}_{s}^{f-i n-1 *}=\left\{\left(R_{1}, R_{2}\right): R_{1} \leq 2-h(p)-h(q)\right. \\
& R_{2} \leq 2-h(p)-h(r) \\
& \left.R_{1}+R_{2} \leq 3-h(p)-h(q)-h(r)\right\} \\
& \mathcal{C}_{s}^{f-\text { in-2 }}=\left\{\left(R_{1}, R_{2}\right): R_{1} \leq 1, R_{2} \leq 1\right. \\
& R_{1} \leq[2-h(p)-h(q)-h(r)]^{+} \\
& \left.R_{2} \leq[2-h(p)-h(q)-h(r)]^{+}\right\} \\
& \mathcal{C}_{s}^{f-\text { out }}=\left\{\left(R_{1}, R_{2}\right): R_{1} \leq 2-h(p)-h(q)\right. \\
& R_{2} \leq 2-h(p)-h(r) \\
& \left.R_{1}+R_{2} \leq 3-h(p)-h(q)-h(r)\right\}
\end{aligned}
$$

where $h(a)=-a \log (a)-(1-a) \log (1-a)\left(0 \leq a \leq \frac{1}{2}\right)$. It is easy to see that if the noises are independent of one another, the achievable secrecy rate region $\mathcal{C}_{s}^{f-i n-1 *}$ of the enhanced secret key based feedback scheme meets the outer bound, which indicates that the secrecy capacity region is determined. The following Figures 5 and 6 plot the above bounds for several values of $p, q$ and $r$. From these two figures, we see that the new inner bound $\mathcal{C}_{s}^{f-i n-2}$ is strictly smaller than the enhanced secret key based inner bound. Moreover, as we see, the new inner bound $\mathcal{C}_{s}^{f-i n-2}$ is decreasing while $p$, $q$ and $r$ are increasing.

B. A special case of Dueck-type example with $Z_{0} \rightarrow Z_{1} \rightarrow Z_{2}$

For the case that $Z_{0} \rightarrow Z_{1} \rightarrow Z_{2}$, define $Z_{0} \sim \operatorname{Bern}(p)$, then $Z_{1}$ is generated from a DMC with channel input $Z_{0}$ and channel transition probability $q$, and $Z_{2}$ is generated from another DMC with channel input $Z_{1}$ and channel transition probability $r$. For this case, the secret key based inner bound $\mathcal{C}_{s f}^{i n-1}$, the enhanced secret key based inner bound $\mathcal{C}_{s f}^{i n-1-n e w}$,

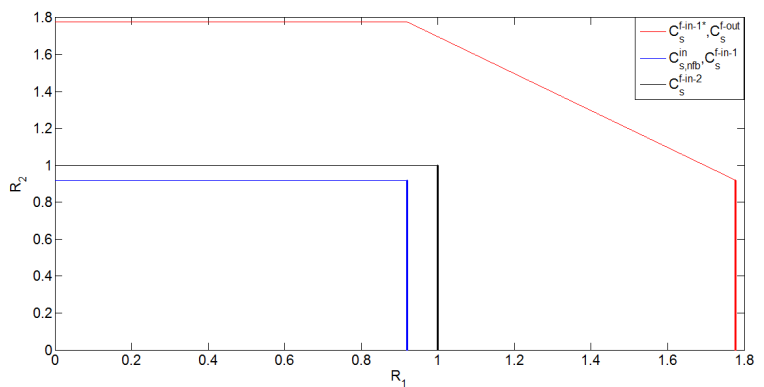

Fig. 5: Comparison of the bounds for $p=0.02, q=0.01$ and $r=0.01$

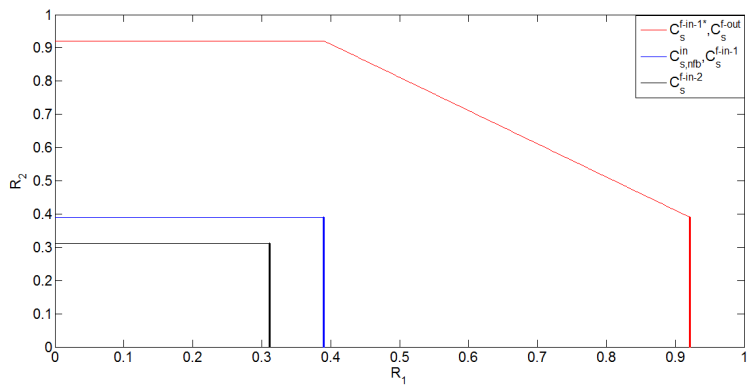

Fig. 6: Comparison of the bounds for $p=0.1, q=0.15$ and $r=0.15$

the new inner bound $\mathcal{C}_{s f}^{i n-2}$, the outer bound $\mathcal{C}_{s f}^{\text {out }}$ and the achievable secrecy rate region $\mathcal{C}_{s}^{i n}$ for the non-feedback model are given by

$$
\begin{aligned}
& \mathcal{C}_{s}^{f-i n-1}=\mathcal{C}_{s, n f b}^{i n}=\left\{\left(R_{1}, R_{2}\right): 0 \leq R_{1} \leq 1-h(q),\right. \\
& \left.0 \leq R_{2} \leq 1-h(q \star r)\right\} \\
& \mathcal{C}_{s}^{f-i n-1 *}=\left\{\left(R_{1}, R_{2}\right): R_{1} \leq 2-h(p)-h(p \star q),\right. \\
& R_{2} \leq 2-h(p)-h((p \star q) \star r), \\
& \left.R_{1}+R_{2} \leq 3-h(p)-h(p \star q)-h((p \star q) \star r)\right\} \\
& \mathcal{C}_{s}^{f-\text { in-2 }}=\left\{\left(R_{1}, R_{2}\right): R_{1} \leq 1, R_{2} \leq 1,\right. \\
& R_{1} \leq[2-h(p)-h(q)-h(r)]^{+} \\
& \left.R_{2} \leq[2-h(p)-h(q)-h(r)]^{+}\right\} \\
& \mathcal{C}_{s}^{f-\text { out }}=\left\{\left(R_{1}, R_{2}\right): R_{1} \leq 2-h(p)-h(q),\right. \\
& R_{2} \leq 2-h(p)-h(q \star r), \\
& \left.R_{1}+R_{2} \leq 3-h(p)-h(q)-h(r)\right\}
\end{aligned}
$$

where $a \star b=a(1-b)+(1-a) b$. The following Figures 7 and 8 plot the above bounds for several values of $p, q$ and $r$. From these two figures, we see that both the new inner bound $\mathcal{C}_{s}^{f-i n-2}$ and the enhanced secret key based inner bound have their advantages and disadvantages. Moreover, as we see, the new inner bound $\mathcal{C}_{s}^{f-i n-2}$ is approaching the outer bound while $p \rightarrow 0, q \rightarrow 0.5$ and $r \rightarrow 0$.

The following special case of this $Z_{0} \rightarrow Z_{1} \rightarrow Z_{2}$-type example explains why the new feedback scheme performs better than the secret key based feedback scheme, see details below. If $p=0, q=\frac{1}{2}$ and $r=0$, the special case $Z_{0} \rightarrow Z_{1} \rightarrow Z_{2}$ reduces to the situation that $Z_{1}=Z_{2}=Z \sim \operatorname{Bern}\left(\frac{1}{2}\right)$ and $Z_{0}=$ const, which implies that the two private channels have the same channel noise $Z$, and the common channel is 


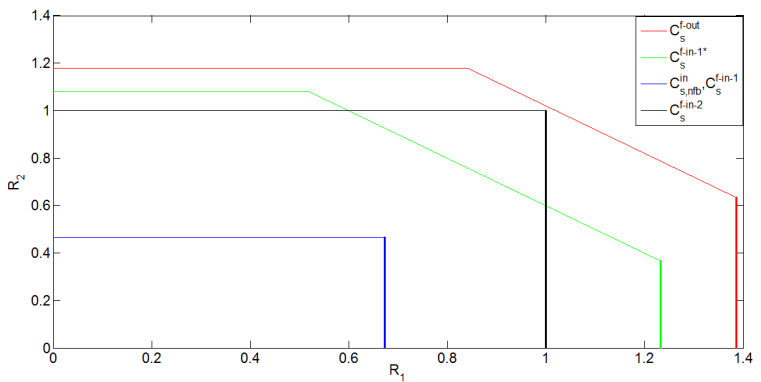

Fig. 7: Comparison of the bounds for the $Z_{0} \rightarrow Z_{1} \rightarrow Z_{2}$ case of the Dueck-type example with $p=0.05, q=0.06$ and $r=0.07$

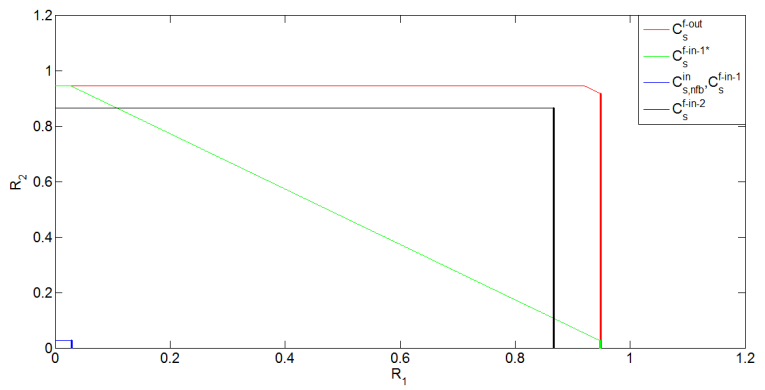

Fig. 8: Comparison of the bounds for the $Z_{0} \rightarrow Z_{1} \rightarrow Z_{2}$ case of the Dueck-type example with $p=0.01, q=0.4$ and $r=0.01$

noiseless, i.e.,

$$
\begin{aligned}
& X=\left(X_{0}, X_{1}, X_{2}\right), Y_{1}=\left(Y_{10}, Y_{11}\right), Y_{2}=\left(Y_{20}, Y_{21}\right), \\
& Y_{10}=Y_{20}=X_{0}, Y_{11}=X_{1} \oplus Z, Y_{21}=X_{2} \oplus Z
\end{aligned}
$$

Now substituting $p=0, q=\frac{1}{2}$ and $r=0$ into (6.2), we get

$$
\begin{aligned}
& \mathcal{C}_{s}^{f-i n-1}=\mathcal{C}_{s, n f b}^{i n}=\left\{\left(R_{1}, R_{2}\right): R_{1}=R_{2}=0\right\}, \\
& \mathcal{C}_{s}^{f-i n-2}=\mathcal{C}_{s}^{f-\text { out }}=\left\{\left(R_{1}, R_{2}\right): R_{1} \leq 1, R_{2} \leq 1\right\}, \\
& \mathcal{C}_{s}^{f-\text { in-1* }}=\left\{\left(R_{1}, R_{2}\right): R_{1}+R_{2} \leq 1\right\} .
\end{aligned}
$$

Here the achievable region $\mathcal{C}_{s}^{f-i n-1 *}$ can be explained as follows. Since the two private channels are completely noisy $\left(Z \sim \operatorname{Bern}\left(\frac{1}{2}\right)\right)$, these two channels cannot be used to transmit messages. The feedback of the two private channels are used to generate secret keys, and these keys protect the messages transmitted over the noiseless common channel. Since the capacity of the common channel equals $1, R_{1}+R_{2} \leq 1$ is achieved.

Moreover, the coding scheme that achieves $\mathcal{C}_{s}^{f-i n-2}$ can be simply described as follows. In block $i(1 \leq i \leq n)$, once receiving the channel output feedback from the two private channels, the noise $Z_{i}$ (i.e., the noise $Z$ of block $i$ ) is completely known by the transmitter due to the reason that $Z_{i}=Y_{11, i} \oplus X_{1, i}$ and $Z_{i}=Y_{21, i} \oplus X_{2, i}$ (here $Y_{11, i}, X_{1, i}, Y_{21, i}$, $X_{2, i}$ represent the $i$-th block's $Y_{11}, X_{1}, Y_{21}, X_{2}$, respectively). Then in block $i+1$, the transmitter sends $X_{0, i+1}=Z_{i}$ through the noiseless common channel, and hence the two receivers obtain the previous time's channel noise $Z_{i}$ of their private channels. Once obtaining $Z_{i}$, the two receivers can respectively compute their channel inputs $X_{1, i}=Y_{11, i} \oplus Z_{i}$ and $X_{2, i}=Y_{21, i} \oplus Z_{i}$, and finally recover the messages transmitted over the private channels. Applying this scheme, it is easy to see that $\mathcal{C}_{s}^{f-i n-2}$ is achieved, i.e., $R_{1} \leq 1$ and $R_{2} \leq 1$. From the above statement, we see that the secret key based feedback scheme is not always optimal, and sometimes the new feedback scheme can do better.

C. A special case of Dueck-type example with $Z_{1} \rightarrow Z_{0} \rightarrow Z_{2}$

For the case that $Z_{1} \rightarrow Z_{0} \rightarrow Z_{2}$, define $Z_{0} \sim \operatorname{Bern}(p)$, then $Z_{1}$ is generated from a DMC with channel input $Z_{0}$ and channel transition probability $q$, and $Z_{2}$ is generated from another DMC with channel input $Z_{0}$ and channel transition probability $r$. For this case, the secret key based inner bound $\mathcal{C}_{s}^{f-i n-1}$, the enhanced secret key based inner bound $\mathcal{C}_{s}^{f-i n-1 *}$, the new inner bound $\mathcal{C}_{s}^{f-i n-2}$, the outer bound $\mathcal{C}_{s}^{f-o u t}$ and the achievable secrecy rate region $\mathcal{C}_{s, n f b}^{\text {in }}$ for the non-feedback model are given by

$$
\begin{aligned}
& \mathcal{C}_{s}^{f-i n-1}=\mathcal{C}_{s, n f b}^{\text {in }}=\left\{\left(R_{1}, R_{2}\right): 0 \leq R_{1} \leq 1-h(q),\right. \\
& \left.0 \leq R_{2} \leq 1-h(r)\right\} \\
& \mathcal{C}_{s}^{f-i n-1 *}=\left\{\left(R_{1}, R_{2}\right): R_{1} \leq 2-h(p)-h(p \star q)\right. \\
& R_{2} \leq 2-h(p)-h(p \star r) \\
& \left.R_{1}+R_{2} \leq 3-h(p)-h(p \star q)-h(p \star r)\right\} \\
& \mathcal{C}_{s}^{f-\text { in-2 }}=\left\{\left(R_{1}, R_{2}\right): R_{1} \leq 1, R_{2} \leq 1\right. \\
& R_{1} \leq[2-h(p)-h(q)-h(r)]^{+} \\
& \left.R_{2} \leq[2-h(p)-h(q)-h(r)]^{+}\right\} \\
& \mathcal{C}_{s}^{f-\text { out }}=\left\{\left(R_{1}, R_{2}\right): R_{1} \leq 2-h(p)-h(q)\right. \\
& R_{2} \leq 2-h(p)-h(r) \\
& \left.R_{1}+R_{2} \leq 3-h(p)-h(q)-h(r)\right\} .
\end{aligned}
$$

The following Figures 9 and 10 plot the above bounds for several values of $p, q$ and $r$. From these two figures, we see that the new inner bound $\mathcal{C}_{s}^{f-i n-2}$ is strictly smaller than the enhanced secret key based inner bound. Moreover, as we see, the gap between the inner and outer bounds is increasing while $p, q$ and $r$ are increasing.

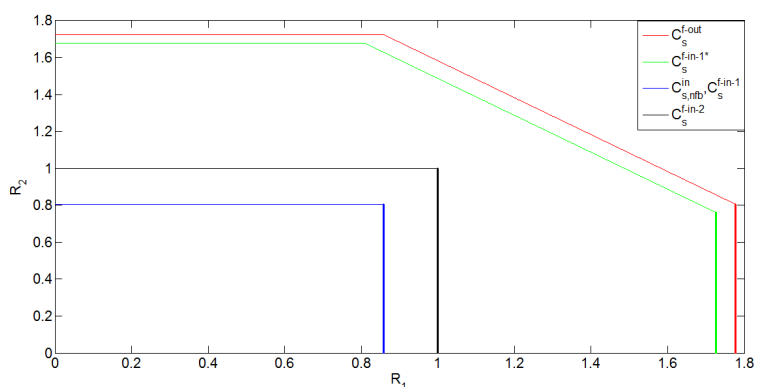

Fig. 9: Comparison of the bounds for the $Z_{1} \rightarrow Z_{0} \rightarrow Z_{2}$ case of the Dueck-type example with $p=0.01, q=0.02$ and $r=0.03$

\section{CONCLUSION}

Two feedback coding strategies for the BC-MSR-R were proposed, and the comparison of the two strategies is shown via a Dueck-type example. From this example, we see that 


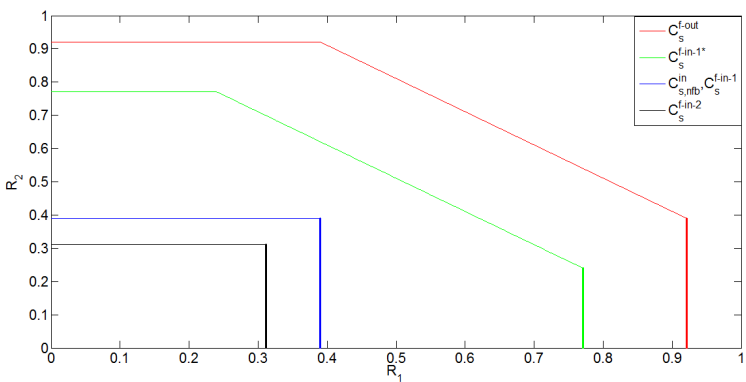

Fig. 10: Comparison of the bounds for the $Z_{1} \rightarrow Z_{0} \rightarrow Z_{2}$ case of the Dueck-type example with $p=0.1, q=0.15$ and $r=0.15$

both the new strategy and the secret key based strategy (including traditional secret key based strategy and the enhanced secret key based strategy) have their advantages and disadvantages, and both strategies achieve the secrecy capacity region of the BC-MSR-R with noiseless feedback for some special cases. The proposed feedback strategies offer new options for enhancing the physical layer security of the broadcast channel models.

\section{APPENDIX A}

\section{PROOF OF THEOREM 2}

The messages are conveyed to the receivers via $n$ blocks. The blocklength of block $i(i \in\{1,2, \ldots, n-1\})$ is $N$, and for block $n$, its blocklength is $\gamma N$, where $\gamma$ is a positive real number and will be determined later. In block $i$ $(i \in\{1,2, \ldots, n-1\})$, the random sequences $X^{N}, Y_{1}^{N}, Y_{2}^{N}$, $Q^{N}, U_{1}^{N}, U_{2}^{N}, V_{0}^{N}, V_{1}^{N}$ and $V_{2}^{N}$ are denoted by $\bar{X}_{i}, \bar{Y}_{1, i}$, $\bar{Y}_{2, i}, \bar{Q}_{i}, \bar{U}_{1, i}, \bar{U}_{2, i}, \bar{V}_{0, i}, \bar{V}_{1, i}$ and $\bar{V}_{2, i}$, respectively. Similarly, in block $n$, the random sequences $X^{\gamma N}, Y_{1}^{\gamma N}, Y_{2}^{\gamma N}, Q^{\gamma N}$, $U_{1}^{\gamma N}$ and $U_{2}^{\gamma N}$ are denoted by $\bar{X}_{n}, \bar{Y}_{1, n}, \bar{Y}_{2, n}, \bar{Q}_{n}, \bar{U}_{1, n}$ and $\bar{U}_{2, n}$, respectively. In addition, the value of the random vector is written in lower case letter.

Code-books generation: The message $W_{j}(j=1,2)$ is sent to Receiver $j$ via $n$ blocks, i.e., the message $W_{j}$ is composed of $n$ components $\left(W_{j}=\left(W_{j, 1}, \ldots, W_{j, n}\right)\right)$, and each component $W_{j, i}(i \in\{1,2, \ldots, n\})$ is the message transmitted in block $i$. Here $W_{j, i}$ takes values in the set $\left\{1, \ldots, 2^{N R_{j}}\right\}$. Further define $W_{j, i}$ as $W_{j, i}=\left(W_{j, 1, i}, W_{j, 2, i}\right)$, where $W_{j, 1, i}$ and $W_{j, 2, i}$ take values in the sets $\left\{1, \ldots, 2^{N R_{j 1}}\right\}$ and $\left\{1, \ldots, 2^{N R_{j 2}}\right\}$, respectively. Here we have $R_{j 1}+R_{j 2}=R_{j}$.

In block $i \quad(1 \leq i \leq n)$, randomly generate $2^{N\left(R_{0}+\tilde{R}_{0}\right)}$ i.i.d. $\bar{Q}_{i}$ w.r.t. $P(q)$, and index them as $\bar{q}_{i}\left(w_{0, i}, w_{0,0, i}^{*}, w_{0,1, i}^{*}, w_{0,2, i}^{*}\right)$, where $w_{0, i} \in\left\{1,2, \ldots, 2^{N R_{0}}\right\}$, $w_{0,0, i}^{*} \in\left\{1,2, \ldots, 2^{N \tilde{R}_{00}}\right\}, w_{0,1, i}^{*} \in\left\{1,2, \ldots, 2^{N \tilde{R}_{01}}\right\}, w_{0,2, i}^{*} \in$ $\left\{1,2, \ldots, 2^{N \tilde{R}_{02}}\right\}$, and $\tilde{R}_{00}+\tilde{R}_{01}+\tilde{R}_{02}=\tilde{R}_{0}$. For each possible value of $\bar{q}_{i}\left(w_{0, i}, w_{0,0, i}^{*}, w_{0,1, i}^{*}, w_{0,2, i}^{*}\right)$, randomly generate $2^{N\left(R_{j}+R_{j}^{\prime}+R_{j}^{\prime \prime}+\tilde{R}_{j}\right)}$ i.i.d. $\bar{U}_{j, i}$ w.r.t. $P\left(u_{j} \mid q\right)$, and index them as $\bar{u}_{j, i}\left(w_{0, i}, w_{0,0, i}^{*}, w_{0,1, i}^{*}, w_{0,2, i}^{*}, w_{j, 1, i}, w_{j, 2, i}, w_{j, i}^{\prime}, w_{j, i}^{\prime \prime}, w_{j, 0, i}^{*}\right.$, $\left.w_{j, j, i}^{*}\right)$, where $j \in\{1,2\}, w_{j, 1, i} \in\left\{1,2, \ldots, 2^{N R_{j 1}}\right\}$, $w_{j, 2, i} \in\left\{1,2, \ldots, 2^{N R_{j 2}}\right\}, w_{j, i}^{\prime} \in\left\{1,2, \ldots, 2^{N R_{j}^{\prime}}\right\}, w_{j, i}^{\prime \prime} \in$ $\left\{1,2, \ldots, 2^{N R_{j}^{\prime \prime}}\right\}, \quad w_{j, 0, i}^{*} \in\left\{1,2, \ldots, 2^{N \tilde{R}_{j 0}}\right\}, \quad w_{j, j, i}^{*} \in$ $\left\{1,2, \ldots, 2^{N \tilde{R}_{j j}}\right\}, R_{j 1}+R_{j 2}=R_{j}$ and $\tilde{R}_{j 0}+\tilde{R}_{j j}=\tilde{R}_{j}$. In block $i$, for each possible values of $\bar{q}_{i}, \bar{u}_{1, i}$ and $\bar{u}_{2, i}$, the channel input $\tilde{X}_{i}$ is i.i.d. generated w.r.t. $P\left(x \mid q, u_{1}, u_{2}\right)$.

For each possible value of $\bar{q}_{i}, \bar{u}_{1, i}, \bar{u}_{2, i}, \bar{y}_{1, i}$ and $\bar{y}_{2, i}$, produce $\bar{V}_{0, i}$ in two ways: 1) Produce $2^{N\left(\tilde{R}_{00}+\tilde{R}_{0}^{\prime}\right)}$ i.i.d. $\bar{V}_{0, i}$ w.r.t. $P\left(v_{0} \mid q, u_{1}, u_{2}, y_{1}, y_{2}\right)$, and index them as $\bar{v}_{0, i}\left(1 ; w_{0,0, i}^{*}, w_{1,0, i}^{*}, t_{1,0, i}\right)$, where $w_{0,0, i}^{*} \in\left\{1,2, \ldots, 2^{N \tilde{R}_{00}}\right\}$, $w_{1,0, i}^{*} \in\left\{1,2, \ldots, 2^{N \tilde{R}_{10}}\right\}$ and $t_{1,0, i} \in\left\{1,2, \ldots, 2^{N\left(\tilde{R}_{0}^{\prime}-\tilde{R}_{10}\right)}\right\}$. 2) Produce $2^{N\left(\tilde{R}_{00}+\tilde{R}_{0}^{\prime}\right)}$ i.i.d. $\bar{V}_{0, i}$ w.r.t. $P\left(v_{0} \mid q, u_{1}, u_{2}, y_{1}, y_{2}\right)$, and index them as $\bar{v}_{0, i}\left(2 ; w_{0,0, i}^{*}, w_{2,0, i}^{*}, t_{2,0, i}\right)$, where $w_{0,0, i}^{*} \in$ $\left\{1,2, \ldots, 2^{N \tilde{R}_{00}}\right\}, w_{2,0, i}^{*} \in\left\{1,2, \ldots, 2^{N \tilde{R}_{20}}\right\}$ and $t_{2,0, i} \in$ $\left\{1,2, \ldots, 2^{N\left(\tilde{R}_{0}^{\prime}-\tilde{R}_{20}\right)}\right\}$.

For each possible value of $\bar{q}_{i}, \bar{u}_{1, i}, \bar{u}_{2, i}, \bar{y}_{1, i}$ and $\bar{y}_{2, i}$, produce $2^{N\left(\tilde{R}_{0 j}+\tilde{R}_{j j}+\tilde{R}_{j}^{\prime}\right)}$ i.i.d. $\bar{V}_{j, i}$ w.r.t. $P\left(v_{j} \mid q, u_{1}, u_{2}, y_{1}, y_{2}\right)$, and index them as $\bar{v}_{j, i}\left(w_{0, j, i}^{*}, w_{j, j, i}^{*}, t_{j, i}\right)$, where $j \in\{1,2\}$, $w_{0, j, i}^{*} \in\left\{1,2, \ldots, 2^{N \tilde{R}_{0 j}}\right\}, w_{j, j, i}^{*} \in\left\{1,2, \ldots, 2^{N \tilde{R}_{j j}}\right\}$ and $t_{j, i} \in\left\{1,2, \ldots, 2^{N \tilde{R}_{j}^{\prime}}\right\}$.

Encoding procedure: At block 1, the transmitter selects $\bar{q}_{1}\left(w_{0,1}, 1,1,1\right), \quad \bar{u}_{1,1}\left(w_{0,1}, 1,1,1, w_{1,1,1}, w_{1,2,1}=\right.$ $\left.1, w_{1,1}^{\prime}, w_{1,1}^{\prime \prime}, 1,1\right) \quad$ and $\quad \bar{u}_{2,1}\left(w_{0,1}, 1,1,1, w_{2,1,1}, w_{2,2,1}=\right.$ $\left.1, w_{2,1}^{\prime}, w_{2,1}^{\prime \prime}, 1,1\right)$. Here notice that $w_{1,1}^{\prime}$ and $w_{2,1}^{\prime}$ are randomly chosen from the sets $\left\{1,2, \ldots, 2^{N R_{1}^{\prime}}\right\}$ and $\left\{1,2, \ldots, 2^{N R_{2}^{\prime}}\right\}$, respectively, and the indexes $w_{1,1}^{\prime \prime}$ and $w_{2,1}^{\prime \prime}$ are chosen by finding a pair of $\left(\bar{u}_{1,1}, \bar{u}_{2,1}\right)$ satisfying the condition that given $\bar{q}_{1},\left(\bar{u}_{1,1}, \bar{u}_{2,1}, \bar{q}_{1}\right)$ are jointly typical. If multiple pairs exist, select the pair with the smallest indexes; if there is no such pair, an encoding error occurs. According to the covering lemma [33], this kind of encoding error tends to zero if

$$
R_{1}^{\prime \prime}+R_{2}^{\prime \prime} \geq I\left(U_{1} ; U_{2} \mid Q\right)
$$

At block $i(i \in\{2,3, \ldots, n-1\})$, before selecting $\bar{u}_{1, i}$ and $\bar{u}_{2, i}$, generate two mappings $g_{1, i}: \bar{y}_{1, i-1} \rightarrow\left\{1,2, \ldots, 2^{N R_{12}}\right\}$ and $g_{2, i}: \bar{y}_{2, i-1} \rightarrow\left\{1,2, \ldots, 2^{N R_{22}}\right\}$. According to these two mappings, generate two RVs $K_{1, i}=g_{1, i}\left(\bar{Y}_{1, i-1}\right)$ and $K_{2, i}=g_{2, i}\left(\bar{Y}_{2, i-1}\right)$ respectively taking values in $\left\{1,2, \ldots, 2^{N R_{12}}\right\}$ and $\left\{1,2, \ldots, 2^{N R_{22}}\right\}$. Here $\operatorname{Pr}\left\{K_{1, i}=j\right\}=2^{-N R_{12}}$ for $j \in\left\{1,2, \ldots, 2^{N R_{12}}\right\}$, and $\operatorname{Pr}\left\{K_{2, i}=l\right\}=2^{-N R_{22}}$ for $l \in\left\{1,2, \ldots, 2^{N R_{22}}\right\}$. The RVs $K_{1, i}$ and $K_{2, i}$ are secret keys encrypting the messages $w_{1,2, i}$ and $w_{2,2, i}$, respectively, and they are independent of the messages $w_{1,2, i}$ and $w_{2,2, i}$. The mappings $g_{1, i}$ and $g_{2, i}$ are revealed to all parties. For $j \in\{1,2\}$, once the transmitter gets $\bar{y}_{j, i-1}$, he seeks a pair of $\left(\bar{v}_{0, i-1}, \bar{v}_{j, i-1}\right)$ satisfying the condition that $\left(\bar{v}_{0, i-1}\left(j ; \tilde{w}_{0,0, i-1}^{*}, \tilde{w}_{j, 0, i-1}^{*}, \tilde{t}_{j, 0, i-1}\right), \bar{v}_{j, i-1}\left(\tilde{w}_{0, j, i-1}^{*}, \tilde{w}_{j, j, i-1}^{*}\right.\right.$, $\left.\left.\tilde{t}_{j, i-1}\right), \bar{u}_{1, i-1}, \bar{u}_{2, i-1}, \bar{q}_{i-1}, \bar{y}_{1, i-1}, \bar{y}_{2, i-1}\right)$ are jointly typical. If there is more than one pair $\left(\bar{v}_{0, i-1}, \bar{v}_{j, i-1}\right)$, randomly choose one pair; if there is no such pair $\left(\bar{v}_{0, i-1}, \bar{v}_{j, i-1}\right)$, an encoding error occurs. From the covering lemma [33], this kind of encoding error tends to zero if

$$
\begin{aligned}
& \tilde{R}_{00}+\tilde{R}_{0}^{\prime} \geq I\left(V_{0} ; Q, U_{1}, U_{2}, Y_{1}, Y_{2}\right), \\
& \tilde{R}_{0 j}+\tilde{R}_{j j}+\tilde{R}_{j}^{\prime} \geq I\left(V_{j} ; V_{0}, Q, U_{1}, U_{2}, Y_{1}, Y_{2}\right) .
\end{aligned}
$$

Once the transmitter selects the pair $\left(\bar{v}_{0, i-1}, \bar{v}_{j, i-1}\right)$, the sequences $\bar{q}_{i}\left(w_{0, i}, \tilde{w}_{0,0, i-1}^{*}, \tilde{w}_{0,1, i-1}^{*}, \tilde{w}_{0,2, i-1}^{*}\right)$ and $\bar{u}_{j, i}\left(w_{0, i}, \tilde{w}_{0,0, i-1}^{*}, \tilde{w}_{0,1, i-1}^{*}, \tilde{w}_{0,2, i-1}^{*}, w_{j, 1, i}, w_{j, 2, i}\right.$ $\left.k_{j, i}, w_{j, i}^{\prime}, w_{j, i}^{\prime \prime}, \tilde{w}_{j, 0, i-1}^{*}, \tilde{w}_{j, j, i-1}^{*}\right) \quad$ are chosen for transmission. Here note that $w_{j, i}^{\prime}, w_{j, i}^{\prime \prime}$ are selected 
the same as those in block 1 . At block $n$, once the transmitter receives the feedback $\bar{y}_{j, n-1}$, he seeks a pair of $\left(\bar{v}_{0, n-1}, \bar{v}_{j, n-1}\right)$ satisfying the condition that $\left(\bar{v}_{0, n-1}\left(j ; \tilde{w}_{0,0, n-1}^{*}, \tilde{w}_{j, 0, n-1}^{*}, \tilde{t}_{j, 0, n-1}\right), \bar{v}_{j, n-1}\left(\tilde{w}_{0, j, n-1}^{*}\right.\right.$, $\left.\left.\tilde{w}_{j, j, n-1}^{*}, \tilde{t}_{j, n-1}\right), \bar{u}_{1, n-1}, \bar{u}_{2, n-1}, \bar{q}_{n-1}, \bar{y}_{1, n-1}, \bar{y}_{2, n-1}\right)$

are jointly typical, and the corresponding encoding error tends to zero when (A2) holds. Finally the transmitter selects $\quad \bar{q}_{n}\left(1, \tilde{w}_{0,0, n-1}^{*}, \tilde{w}_{0,1, n-1}^{*}, \tilde{w}_{0,2, n-1}^{*}\right)$, $\bar{u}_{j, n}\left(1, \tilde{w}_{0,0, n-1}^{*}, \tilde{w}_{0,1, n-1}^{*}, \tilde{w}_{0,2, n-1}^{*}, 1,1,1,1, \tilde{w}_{j, 0, n-1}^{*}\right.$, $\left.\tilde{w}_{j, j, n-1}^{*}\right)$ to transmit.

Decoding procedure: Receiver $j$ 's $(j=1,2)$ decoding procedure begins from block $n$. At block $n$, first, note that due to the reason that the blocklength is $\gamma N$, the actual rate of $W_{0,0, n-1}^{*}$ is given by $\frac{H\left(W_{0,0, n-1}^{*}\right)}{\gamma}=\frac{\tilde{R}_{00}}{\gamma}$. Analogously, the actual rates of $W_{0,1, n-1}^{*}, W_{0,2, n-1}^{*}, W_{1,0, n-1}^{*}, W_{1,1, n-1}^{*}$, $W_{2,0, n-1}^{*}$ and $W_{2,2, n-1}^{*}$ are $\frac{\tilde{R}_{01}}{\gamma}, \frac{\tilde{R}_{02}}{\gamma}, \frac{\tilde{R}_{10}}{\gamma}, \frac{\tilde{R}_{11}}{\gamma}, \frac{\tilde{R}_{20}}{\gamma}$ and $\frac{\tilde{R}_{22}}{\gamma}$, respectively. Next, Receiver $j$ chooses a unique pair of $\left(\bar{q}_{n}, \bar{u}_{j, n}\right)$ such that $\left(\bar{q}_{n}, \bar{u}_{j, n}, \bar{y}_{j, n}\right)$ are jointly typical. If there is more than one or no such pair, a decoding error occurs. From the packing lemma [33], this kind of decoding error tends to zero if

$$
\begin{aligned}
& \frac{\tilde{R}_{0}}{\gamma} \leq I\left(Q, U_{j} ; Y_{j}\right), \frac{\tilde{R}_{j}}{\gamma} \leq I\left(Y_{j} ; U_{j} \mid Q\right), \\
& \frac{\tilde{R}_{0}}{\gamma}+\frac{\tilde{R}_{j}}{\gamma} \leq I\left(Q, U_{j} ; Y_{j}\right) .
\end{aligned}
$$

Since $I\left(Q, U_{j} ; Y_{j}\right)$ and $I\left(Y_{j} ; U_{j} \mid Q\right)$ of (A3) are finite, for any given $\tilde{R}_{0}$ and $\tilde{R}_{j}$, we can choose a sufficiently large $\gamma$ such that (A3) is satisfied. Once $\bar{q}_{n}$ and $\bar{u}_{j, n}$ are decoded, Receiver $j$ extracts $w_{0,0, n-1}^{*}, w_{0,1, n-1}^{*}, w_{0,2, n-1}^{*}, w_{j, 0, n-1}^{*}$ and $w_{j, j, n-1}^{*}$ in them. Then according to the extracted messages, Receiver $j$ seeks a unique pair of $\left(\bar{v}_{0, n-1}, \bar{v}_{j, n-1}\right)$ satisfying the condition that $\bar{v}_{0, n-1}\left(j ; w_{0,0, n-1}^{*}, w_{j, 0, n-1}^{*}, t_{j, 0, n-1}\right), \bar{v}_{j, n-1}\left(w_{0, j, n-1}^{*}\right.$, $\left.\left.w_{j, j, n-1}^{*}, t_{j, n-1}\right), \bar{y}_{j, n-1}\right)$ are jointly typical. This kind of decoding error tends to zero if

$$
\begin{aligned}
& \tilde{R}_{j}^{\prime} \leq I\left(V_{j} ; V_{0}, Y_{j}\right), \\
& \tilde{R}_{j}^{\prime}+\tilde{R}_{0}^{\prime}-\tilde{R}_{j 0} \leq I\left(V_{0} ; Y_{j}\right)+I\left(V_{j} ; V_{0}, Y_{j}\right) .
\end{aligned}
$$

Here note that (A4) is from the packing lemma and the multivariate packing lemma [33]. Then, for block $n-1$, after $\bar{v}_{0, n-1}$ and $\bar{v}_{j, n-1}$ are decoded, Receiver $j$ chooses a unique pair of $\left(\bar{q}_{n-1}, \bar{u}_{j, n-1}\right)$ such that $\left(\bar{u}_{j, n-1}, \bar{q}_{n-1}, \bar{y}_{j, n-1}, \bar{v}_{j, n-1}\right)$ are jointly typical, and from the packing lemma [33], this kind of decoding error tends to zero if

$$
\begin{aligned}
& R_{0}+\tilde{R}_{0} \leq I\left(Q, U_{j} ; V_{j}, Y_{j}\right), \\
& R_{j 1}+R_{j 2}+R_{j}^{\prime}+R_{j}^{\prime \prime}+\tilde{R}_{j 0}+\tilde{R}_{j, j} \\
& \leq I\left(U_{j} ; V_{j}, Y_{j} \mid Q\right), \\
& R_{0}+\tilde{R}_{0}+R_{j 1}+R_{j 2}+R_{j}^{\prime}+R_{j}^{\prime \prime}+\tilde{R}_{j 0}+\tilde{R}_{j, j} \\
& \leq I\left(Q, U_{j} ; V_{j}, Y_{j}\right) .
\end{aligned}
$$

Here note that (A5) is included in (A7), and thus it is useless. When $\bar{q}_{n-1}$ and $\bar{u}_{j, n-1}$ are decoded, Receiver $j$ extracts $w_{0, n-1}, w_{0,0, n-2}^{*} w_{0,1, n-2}^{*}, w_{0,2, n-2}^{*}, w_{j, 1, n-1}, w_{j, 2, n-1} \oplus$ $k_{j, n-1}, w_{j, n-1}^{\prime}, w_{j, n-1}^{\prime \prime}, w_{j, 0, n-2}^{*}$ and $w_{j, j, n-2}^{*}$. Since the key $k_{j, n-1}$ generated from $\bar{y}_{j, n-2}$ is also known by Receiver $j$, the messages $w_{j, 1, n-1}$ and $w_{j, 2, n-1}$ are correctly decoded by Receiver $j$. Repeat the above decoding procedure, the messages of all blocks are decoded by Receiver $j$.
Equivocation Analysis for Receiver 2: Receiver 2's equivocation $\Delta_{1}$, defined as $\Delta_{1}=\frac{1}{(n-1) N+\gamma N} H\left(W_{1} \mid \bar{Y}_{2,1}, \ldots, Y_{2, n}\right)$, follows that

$$
\begin{aligned}
& \Delta_{1} \stackrel{(a)}{=} \frac{1}{(n-1) N+\gamma N} H\left(\tilde{W}_{11}, \tilde{W}_{12} \mid \bar{Y}_{2,1}, \ldots, \bar{Y}_{2, n}\right) \\
& =\frac{1}{(n-1) N+\gamma N}\left(H\left(\tilde{W}_{11} \mid \bar{Y}_{2,1}, \ldots, \bar{Y}_{2, n}\right)\right. \\
& \left.+H\left(\tilde{W}_{12} \mid \bar{Y}_{2,1}, \ldots, \bar{Y}_{2, n}, \tilde{W}_{11}\right)\right),
\end{aligned}
$$

where (a) follows from the definitions $\tilde{W}_{11}=$ $\left(W_{1,1,1}, \ldots, W_{1,1, n}=1\right)$ and $\tilde{W}_{12}=\left(W_{1,2,1}=\right.$ $\left.1, W_{1,2,2}, \ldots, W_{1,2, n-1}, W_{1,2, n}=1\right)$. The first part $H\left(\tilde{W}_{11} \mid \bar{Y}_{2,1}, \ldots, \bar{Y}_{2, n}\right)$ of (A8) is bounded by

$$
\begin{aligned}
& H\left(\tilde{W}_{11} \mid \bar{Y}_{2,1}, \ldots, \bar{Y}_{2, n}\right) \stackrel{(b)}{=} \sum_{i=1}^{n-1} H\left(W_{1,1, i} \mid \bar{Y}_{2, i}\right) \\
& \geq \sum_{i=1}^{n-1} H\left(W_{1,1, i} \mid \bar{Y}_{2, i}, \bar{Q}_{i}, \bar{U}_{2, i}, \bar{V}_{2, i}\right) \\
& \stackrel{(c)}{=} \sum_{i=1}^{n-1}\left(H\left(\bar{Y}_{2, i}, \bar{V}_{2, i} \mid \bar{Q}_{i}, \bar{U}_{1, i}, \bar{U}_{2, i}\right)+H\left(\bar{U}_{1, i} \mid \bar{Q}_{i}, \bar{U}_{2, i}\right)\right. \\
& \left.-H\left(\bar{U}_{1, i} \mid W_{1,1, i}, \bar{Y}_{2, i}, \bar{Q}_{i}, \bar{U}_{2, i}, \bar{V}_{2, i}\right)-H\left(\bar{Y}_{2, i}, \bar{V}_{2, i} \mid \bar{Q}_{i}, \bar{U}_{2, i}\right)\right) \\
& =\sum_{i=1}^{n-1}\left(H\left(\bar{U}_{1, i} \mid \bar{Q}_{i}\right)-I\left(\bar{U}_{1, i} ; \bar{U}_{2, i} \mid \bar{Q}_{i}\right)-I\left(\bar{U}_{1, i} ; \bar{Y}_{2, i}, \bar{V}_{2, i} \mid \bar{Q}_{i}, \bar{U}_{2, i}\right)\right. \\
& \left.-H\left(\bar{U}_{1, i} \mid W_{1,1, i}, \bar{Y}_{2, i}, \bar{Q}_{i}, \bar{U}_{2, i}, \bar{V}_{2, i}\right)\right) \\
& \stackrel{(d)}{\geq}\left(R_{11}+R_{1}^{\prime}+R_{1}^{\prime \prime}+\tilde{R}_{10}+\tilde{R}_{11}\right)-N \epsilon_{6} \\
& +\sum_{i=2}^{n-1}\left(N\left(R_{11}+R_{12}+R_{1}^{\prime}+R_{1}^{\prime \prime}+\tilde{R}_{10}+\tilde{R}_{11}\right)-N \epsilon_{7}\right) \\
& -(n-1) N\left(I\left(U_{1} ; U_{2} \mid Q\right)+I\left(U_{1} ; Y_{2}, V_{2} \mid Q, U_{2}\right)+\epsilon_{4}+\epsilon_{5}\right) \\
& -\sum_{i=1}^{n-1} H\left(\bar{U}_{1, i} \mid W_{1,1, i}, \bar{Y}_{2, i}, \bar{Q}_{i}, \bar{U}_{2, i}, \bar{V}_{2, i}\right) \\
& (e) \\
& \geq N\left(R_{11}+R_{1}^{\prime}+R_{1}^{\prime \prime}+\tilde{R}_{10}+\tilde{R}_{11}-\epsilon_{6}-\epsilon_{9}\right)-(n-2) N \epsilon_{8} \\
& +(n-2) N\left(R_{11}+R_{12}+R_{1}^{\prime}+R_{1}^{\prime \prime}+\tilde{R}_{10}+\tilde{R}_{11}-\epsilon_{7}\right) \\
& -(n-1) N\left(I\left(U_{1} ; U_{2} \mid Q\right)+I\left(U_{1} ; Y_{2}, V_{2} \mid Q, U_{2}\right)+\epsilon_{4}+\epsilon_{5}\right), \quad(\mathrm{A} 9) \\
& +(n)
\end{aligned}
$$

where (b) follows from the Markov chain $W_{1,1, i} \rightarrow$ $\bar{Y}_{2, i} \rightarrow\left(W_{1,1,1}, \ldots, W_{1,1, i-1}, \bar{Y}_{2,1}, \ldots, \bar{Y}_{2, i-1}, \bar{Y}_{2, i+1}, \ldots, \bar{Y}_{2, n}\right)$ holds, (c) follows from $H\left(\tilde{W}_{1,1, i} \mid \bar{U}_{1, i}\right)=0$, (d) follows from a similar argument in [3, Lemma 3] (i.e., $I\left(\bar{U}_{1, i} ; \bar{U}_{2, i} \mid \bar{Q}_{i}\right) \leq N\left(I\left(U_{1} ; U_{2} \mid Q\right)+\epsilon_{4}\right)\left(\epsilon_{4} \rightarrow 0\right.$ as $N \rightarrow$ $\infty), I\left(\bar{U}_{1, i} ; \bar{Y}_{2, i}, \overline{\bar{V}}_{2, i} \mid \bar{Q}_{i}, \bar{U}_{2, i}\right) \leq N\left(I\left(U_{1} ; Y_{2}, V_{2} \mid Q, U_{2}\right)+\epsilon_{5}\right)$ $\left(\epsilon_{5} \rightarrow 0\right.$ as $\left.N \rightarrow \infty\right)$ ), and a similar argument in [2, equations (16) and (23)], i.e., $H\left(\bar{U}_{1,1} \mid \bar{Q}_{1}\right) \geq N\left(R_{11}+\right.$ $\left.R_{1}^{\prime}+R_{1}^{\prime \prime}+\tilde{R}_{10}+\tilde{R}_{11}\right)-N \epsilon_{6}\left(\epsilon_{6} \rightarrow 0\right.$ as $\left.N \rightarrow \infty\right)$, $H\left(\bar{U}_{1, i} \mid \bar{Q}_{i}\right) \geq N\left(R_{11}+R_{12}+R_{1}^{\prime}+R_{1}^{\prime \prime}+\tilde{R}_{10}+\tilde{R}_{11}\right)-N \epsilon_{7}$ for $i \in\{2, \ldots, n-1\}\left(\epsilon_{7} \rightarrow 0\right.$ as $\left.N \rightarrow \infty\right)$, and (e) follows from that given $w_{1,1,1}, \bar{y}_{2,1}, \bar{q}_{1}, \bar{u}_{2,1}, \bar{v}_{2,1}$, Receiver 2 seeks a unique $\bar{u}_{1,1}$ that is jointly typical with his/her own received signals $\bar{y}_{2,1}, \bar{q}_{1}, \bar{u}_{2,1}, \bar{v}_{2,1}$, and from the packing lemma [33], this decoding error tends to zero if

$$
R_{1}^{\prime}+R_{1}^{\prime \prime}+\tilde{R}_{1}+\leq I\left(Y_{2}, V_{2} ; U_{1} \mid Q, U_{2}\right),
$$

$H\left(\bar{U}_{1,1} \mid W_{1,1,1}, \bar{Y}_{2,1}, \bar{Q}_{1}, \bar{U}_{2,1}, \bar{V}_{2,1}\right) \leq N \epsilon_{9}$ is obtained by Fano's lemma, where $\epsilon_{9} \rightarrow 0$ while $\bar{N} \rightarrow \infty$, and similarly, for $i \in\{2, \ldots, n-1\}$ given $w_{1,1, i}, \bar{y}_{2, i}, \bar{q}_{i}, \bar{u}_{2, i}, \bar{v}_{2, i}$, Receiver 2 seeks a unique $\bar{u}_{1, i}$ that is jointly typical with his/her own received signals $\bar{y}_{2, i}, \bar{q}_{i}, \bar{u}_{2, i}, \bar{v}_{2, i}$, and from the packing lemma 
[33], this decoding error tends to zero if

$$
R_{12}+R_{1}^{\prime}+R_{1}^{\prime \prime}+\tilde{R}_{1} \leq I\left(Y_{2}, V_{2} ; U_{1} \mid Q, U_{2}\right)
$$

$H\left(\bar{U}_{1, i} \mid W_{1,1, i}, \bar{Y}_{2, i}, \bar{Q}_{i}, \bar{U}_{2, i}, \bar{V}_{2, i}\right) \leq N \epsilon_{8}$ is obtained by Fano's lemma, where $\epsilon_{8} \rightarrow 0$ while $N \rightarrow \infty$. Here note that (A10) is included in (A11), and thus we only need to use (A11) to derive the final region of this achievability scheme.

The second part of (A8) is bounded by,

$$
\begin{aligned}
& H\left(\tilde{W}_{12} \mid \bar{Y}_{2,1}, \ldots, \bar{Y}_{2, n}, \tilde{W}_{11}\right) \\
\geq & \sum_{i=2}^{n-1} H\left(W_{1,2, i} \mid \bar{Y}_{2,1}, \ldots, \bar{Y}_{2, n}, \tilde{W}_{11}, W_{1,2,1}=1, \ldots,\right. \\
& \left.W_{1,2, i-1}, W_{1,2, i} \oplus K_{1, i}\right) \\
& \stackrel{(f)}{=} \sum_{i=2}^{n-1} H\left(W_{1,2, i} \mid \bar{Y}_{2, i-1}, W_{1,2, i} \oplus K_{1, i}\right) \\
\geq & \sum_{i=2}^{n-1} H\left(W_{1,2, i} \mid \bar{Y}_{2, i-1}, W_{1,2, i} \oplus K_{1, i}, \bar{V}_{2, i-1}, \bar{Q}_{i-1},\right. \\
& \left.\bar{U}_{1, i-1}, \bar{U}_{2, i-1}\right) \\
\underline{(g)} & \sum_{i=2}^{n-1} H\left(K_{1, i} \mid \bar{Y}_{2, i-1}, \bar{V}_{2, i-1}, \bar{Q}_{i-1}, \bar{U}_{1, i-1}, \bar{U}_{2, i-1}\right) \\
(h) & (n-2)\left(\log \frac{1-\epsilon_{1}}{1+\delta}\right. \\
& \left.+N\left(1-\epsilon_{2}\right) H\left(Y_{1} \mid Y_{2}, V_{2}, Q, U_{1}, U_{2}\right)\right),
\end{aligned}
$$

where (f) follows from the Markov chain $W_{1,2, i} \rightarrow$ $\left(\bar{Y}_{2, i-1}, W_{1,2, i} \oplus K_{1, i}\right) \rightarrow\left(\tilde{W}_{11}, W_{1,2,1}, \ldots, W_{1,2, i-1}, \bar{Y}_{2,1}\right.$, $\left.\ldots, \bar{Y}_{2, i-2}, \bar{Y}_{2, i}, \ldots, \bar{Y}_{2, n}\right), \quad$ (g) follows from $K_{1, i} \rightarrow$ $\left(\bar{Y}_{2, i-1}, \bar{V}_{2, i-1}, \bar{Q}_{i-1}, \bar{U}_{1, i-1}, \bar{U}_{2, i-1}\right) \quad \rightarrow \quad W_{1,2, i} \oplus K_{1, i}$, and (h) follows from Lemma 3 in [27, p.264].

Finally, substituting (A12) and (A9) into (A8), we can conclude that if

$$
\begin{aligned}
& R_{1}^{\prime}+R_{1}^{\prime \prime}+\tilde{R}_{10}+\tilde{R}_{11} \geq I\left(U_{1} ; U_{2} \mid Q\right)+I\left(V_{2}, Y_{2} ; U_{1} \mid Q, U_{2}\right) \\
& -H\left(Y_{1} \mid Y_{2}, V_{2}, Q, U_{1}, U_{2}\right),
\end{aligned}
$$

$\Delta_{1} \geq R_{1}-\epsilon$ is satisfied by choosing sufficiently large $n$ and $N$.

Equivocation Analysis for Receiver 1: The equivocation analysis of Receiver 1's equivocation $\Delta_{2}=$ $\frac{1}{(n-1) N+\gamma N} H\left(W_{2} \mid \bar{Y}_{1,1}, \ldots, \bar{Y}_{1, n}\right)$ is analogous to that of $\Delta_{1}$. Hence choosing sufficiently large $n$ and $N, \Delta_{2} \geq R_{2}-\epsilon$ can be proved if

$$
\begin{aligned}
& R_{22}+R_{2}^{\prime}+R_{2}^{\prime \prime}+\tilde{R}_{20}+\tilde{R}_{22} \\
& \leq I\left(Y_{1}, V_{1} ; U_{2} \mid Q, U_{1}\right), \\
& R_{2}^{\prime}+R_{2}^{\prime \prime}+\tilde{R}_{20}+\tilde{R}_{22} \geq I\left(U_{1} ; U_{2} \mid Q\right) \\
& +I\left(V_{1}, Y_{1} ; U_{2} \mid Q, U_{1}\right)-H\left(Y_{2} \mid Y_{1}, V_{1}, Q, U_{1}, U_{2}\right) . \text { (A15) }
\end{aligned}
$$

Fourier-Motzkin elimination: First, using the fact that $\tilde{R}_{0}=$ $\tilde{R}_{00}+\tilde{R}_{01}+\tilde{R}_{02}, \tilde{R}_{1}=\tilde{R}_{10}+\tilde{R}_{11}, \tilde{R}_{2}=\tilde{R}_{20}+\tilde{R}_{22}, \tilde{X}=$ $\left(Q, U_{1}, U_{2}, Y_{1}, Y_{2}\right)$, and applying Fourier-Motzkin elimination to remove $\tilde{R}_{0}^{\prime}, \tilde{R}_{1}^{\prime}$ and $\tilde{R}_{2}^{\prime}$ from (A2) and (A4), we have

$$
\begin{aligned}
& \tilde{R}_{0}+\tilde{R}_{1} \geq I\left(V_{0}, V_{1} ; \tilde{X} \mid Y_{1}\right), \tilde{R}_{0}+\tilde{R}_{2} \geq I\left(V_{0}, V_{2} ; \tilde{X} \mid Y_{2}\right) \\
& \tilde{R}_{0}+\tilde{R}_{1}+\tilde{R}_{2} \geq I\left(V_{1} ; \tilde{X} \mid Y_{1}, V_{0}\right)+I\left(V_{2} ; \tilde{X} \mid Y_{2}, V_{0}\right) \\
& +\max \left\{I\left(V_{0} ; \tilde{X} \mid Y_{1}\right), I\left(V_{0} ; \tilde{X} \mid Y_{2}\right)\right\} .
\end{aligned}
$$

Next, using $R_{1}=R_{11}+R_{12}$ and $R_{2}=R_{21}+R_{22}$, applying Fourier-Motzkin elimination to remove $R_{1}^{\prime}, R_{1}^{\prime \prime}, R_{2}^{\prime}, R_{2}^{\prime}, \tilde{R}_{0}$,
$\tilde{R}_{1}$ and $\tilde{R}_{2}$ from (A16), (A1), (A6), (A7), (A11), (A13), (A14) and (A15), and using the following conditions

$$
\begin{aligned}
& I\left(U_{1} ; V_{1}, Y_{1} \mid Q\right)+I\left(U_{2} ; V_{2}, Y_{2} \mid Q\right) \geq I\left(U_{1} ; U_{2} \mid Q\right), \\
& I\left(U_{1} ; V_{2}, Y_{2} \mid Q, U_{2}\right) \geq I\left(U_{1} ; U_{2} \mid Q\right), \\
& I\left(U_{2} ; V_{1}, Y_{1} \mid Q, U_{1}\right) \geq I\left(U_{1} ; U_{2} \mid Q\right), \\
& \min \left\{H\left(Y_{2} \mid Q, U_{1}, U_{2}, V_{1}, Y_{1}\right), H\left(Y_{1} \mid Q, U_{1}, U_{2}, V_{2}, Y_{2}\right)\right\} \geq \\
& I\left(U_{1} ; V_{2}, Y_{2} \mid Q, U_{2}\right)+I\left(U_{2} ; V_{1}, Y_{1} \mid Q, U_{1}\right)-2 I\left(V_{1} ; \tilde{X} \mid Y_{1}, V_{0}\right) \\
& -I\left(V_{2} ; Q, U_{1}, U_{2}, Y_{1}, Y_{2} \mid Y_{2}, V_{0}\right), \\
& H\left(Y_{2} \mid Q, U_{1}, U_{2}, V_{1}, Y_{1}\right) \geq I\left(U_{2} ; V_{1}, Y_{1} \mid Q, U_{1}\right), \\
& H\left(Y_{1} \mid Q, U_{1}, U_{2}, V_{2}, Y_{2}\right) \geq I\left(U_{1} ; V_{2}, Y_{2} \mid Q, U_{2}\right), \\
& I\left(U_{1} ; U_{2} \mid Q\right)+I\left(V_{1} ; \tilde{X} \mid Y_{1}, V_{0}\right) \geq I\left(U_{1} ; V_{2}, Y_{2} \mid Q, U_{2}\right), \\
& I\left(U_{1} ; U_{2} \mid Q\right)+I\left(V_{2} ; \tilde{X} \mid Y_{2}, V_{0}\right) \geq I\left(U_{2} ; V_{1}, Y_{1} \mid Q, U_{1}\right), \quad(\mathrm{A} 17)
\end{aligned}
$$

the region $\mathcal{C}_{s}^{f-i n-2}$ in Theorem 2 with the conditions in (A17) is obtained. Next, we show that for every choice of $\left(Q, U_{1}, U_{2}, V_{0}, V_{1}, V_{2}, X\right)$ that satisfies the joint distribution (4.1) and does not satisfy one or more conditions in (A17), we can strictly enlarge the rate region $\mathcal{C}_{s}^{f-i n-2}$ if we replace the random triples $\left(Q, U_{1}, U_{2}\right)$ and $\left(V_{0}, V_{1}, V_{2}\right)$ by $\left(Q^{\prime}, U_{1}^{\prime}, U_{2}^{\prime}\right)$ and $\left(V_{0}^{\prime}, V_{1}^{\prime}, V_{2}^{\prime}\right)$, respectively, where $U_{1}^{\prime}, U_{2}^{\prime}, V_{1}^{\prime}, V_{2}^{\prime}$ are constants and $Q^{\prime}=\left(Q, U_{1}, U_{2}\right), V_{0}^{\prime}=\left(V_{0}, V_{1}, V_{2}\right)$. It is easy to check that the new choice $\left(Q^{\prime}, U_{1}^{\prime}, U_{2}^{\prime}, V_{0}^{\prime}, V_{1}^{\prime}, V_{2}^{\prime}, X\right)$ also satisfies the joint distribution (4.1), and all the conditions in (A17) are satisfied since the right hand side of (A17) equals to 0 , and the corresponding left hand side is larger than or equals to 0 . We thus conclude that the rate region in Theorem 2 is achievable also when the constraint conditions (A17) are violated. Finally, note that the rate $R_{j}(j \in\{1,2\})$ in the above obtained region is only the transmission rate of one block, and the overall effective transmission rate $\bar{R}_{j}^{*}$ is given by

$$
\bar{R}_{j}^{*}=\frac{n-1}{n-1+\gamma} R_{j 1}+\frac{n-2}{n-1+\gamma} R_{j 2} .
$$

From (A18), we can conclude that $\bar{R}_{j}^{*}$ approaches $R_{j}=$ $R_{j 1}+R_{j 2}$ as the number of blocks $n \rightarrow \infty$, and the proof of Theorem 2 is completed.

\section{ACKNOWLEDGMENT}

The authors would like to thank the editor and the anonymous reviewers for their valuable suggestions on improving this paper.

\section{REFERENCES}

[1] A. D. Wyner, "The wire-tap channel," The Bell System Technical Journal, vol. 54, no. 8, pp. 1355-1387, August 1975.

[2] I. Csiszár, J. Körner, "Broadcast channels with confidential messages," IEEE Trans. Inf. Theory, vol. IT-24, no. 3, pp. 339-348, May 1978.

[3] R. Liu, I. Maric, P. Spasojević, R.D Yates, "Discrete memoryless interference and broadcast channels with confidential messages: secrecy rate regions," IEEE Trans. Inf. Theory, vol. IT-54, no. 6, pp. 2493-2507, June 2008.

[4] J. Xu, Y. Cao, B. Chen, "Capacity bounds for broadcast channels with confidential messages," IEEE Trans. Inf. Theory, vol. IT-55, no. 6, pp. 4529-4542. June 2009.

[5] E. Ekrem, S. Ulukus, "Secrecy capacity of a class of broadcast channels with an eavesdropper," EURASIP Journal on Wireless Communications and Networking, vol. 2009, no. 1, pp. 1-29, January 2009.

[6] G. Bagherikaram, A. S. Motahari, A. K. Khandani, "The secrecy rate region of the broadcast channel," Proceedings of the Allerton Conference on Communications, Control and Computing, 2008.

[7] E. Tekin, A. Yener, "The Gaussian multiple access wire-tap channel," IEEE Trans. Inf. Theory, vol. IT-54, no. 12, pp. 5747-5755, December 2008. 
[8] B. Dai, Z. Ma, "Multiple-access relay wiretap channel," IEEE Trans. Inf. Forensics and Security, vol. 10, no. 9, pp. 1835-1849, September 2015.

[9] L. Lai, H. El Gamal, "The relay-eavesdropper channel: cooperation for secrecy," IEEE Trans. Inf. Theory, vol. IT-54, no. 9, pp. 4005C4019, September 2008

[10] E. Ekrem and S. Ulukus, "Secrecy in cooperative relay broadcast channels," IEEE Trans. Inf. Theory, vol. IT-57, no. 1, pp. 137-155, January 2011.

[11] B. Dai, L. Yu, Z. Ma, "Relay broadcast channel with confidential messages," IEEE Trans. Inf. Forensics and Security, vol. 11, no. 2, pp. 410-425, February 2016.

[12] Y. Oohama, "Coding for relay channels with confidential messages," in Proceedings of IEEE Information Theory Workshop, Australia, 2001.

[13] Y. Liang, H. V. Poor, "Multiple-access channels with confidential messages," IEEE Trans. Inf. Theory, vol. IT-54, no. 3, pp. 976-1002, March 2008.

[14] E. Tekin, A. Yener, "The general Gaussian multiple access and twoway wire-tap channels: Achievable rates and cooperative jamming," IEEE Trans. Inf. Theory, vol. IT-54, no. 6, pp. 2735-2751, June 2008.

[15] A. El Gamal, O. O. Koyluoglu, M. Youssef and H. El Gamal, "Achievable secrecy rate regions for the two-way wiretap channel," IEEE Trans. Inf. Theory, vol. IT-59, no. 12, pp. 8099-8114, December 2013.

[16] Q. Zhang, X. Huang, Q. Li, J. Qin, "Cooperative jamming aided robust secure transmission for wireless information and power transfer in MISO channels," IEEE Trans. Commun., vol. 63, no. 3, pp. 906-915, March 2015.

[17] G. Zheng, L. C. Choo, K. K. Wong, "Optimal cooperative jamming to enhance physical layer security using relays," IEEE Trans. Signal Process., vol. 59, no. 3, pp. 1317-1322, March 2011.

[18] K. H. Park, T. Wang, M. S. Alouini, "On the jamming power allocation for secure amplify-and-forward relaying via cooperative jamming," IEEE J. Sel. Areas Commun., vol. 31, no. 9, pp. 1741-1750, September 2013.

[19] J. M. Liang, J. J. Chen, H. H. Cheng, Y. C. Tseng, "An energyefficient sleep scheduling with QoS consideration in 3GPP LTE-Advanced networks for Internet of Things, IEEE J. Emerg. Sel. Top. Circuits Syst., vol. 3, no. 1, pp. 13C22, January 2013.

[20] R. Ahlswede, N. Cai, "Transmission, Identification and Common Randomness Capacities for Wire-Tap Channels with Secure Feedback from the Decoder," book chapter in General Theory of Information Transfer and Combinatorics, LNCS 4123, pp. 258-275, Berlin: Springer-Verlag, 2006.

[21] E. Ardestanizadeh, M. Franceschetti, T. Javidi, Y. Kim, "Wiretap channel with secure rate-limited feedback," IEEE Trans. Inf. Theory, vol. IT-55, no. 12 , pp. 5353-5361, December 2009.

[22] R. F. Schaefer, A. Khisti, H. V. Poor, "Secure broadcasting using independent secret keys," IEEE Trans. Commun., vol. 66, no. 2, pp. 644661, February 2018.

[23] A. Cohen, A. Cohen, "Wiretap channel with causal state information and secure rate-limited feedback," IEEE Trans. Commun., vol. 64, no. 3, pp. 1192-1203, March 2016.

[24] B. Dai, Z. Ma, X. Fang, "Feedback enhances the security of statedependent degraded broadcast channels with confidential messages," IEEE Trans. Inf. Forensics and Security, vol. 10, no. 7, pp. 1529-1542, July 2015.

[25] B. Dai, Z. Ma and Y. Luo, "Finite state Markov wiretap channel with delayed feedback," IEEE Trans. Inf. Forensics Security, Vol. 12, no. 3, pp. 746-760, March 2017.

[26] B. Dai, Z. Ma, M. Xiao, X. Tang, P. Fan, "Secure communication over finite state multiple-access wiretap channel with delayed feedback," IEEE J. Sel. Areas Commun., vol. 36, no. 4, pp. 723-736, April 2018.

[27] B. Dai, Y. Luo, "An improved feedback coding scheme for the wiretap channel," IEEE Trans. Inf. Forensics and Security, vol. 14, no. 1, pp. 262271, January 2019.

[28] D. Gunduz, D. R. Brown and H. V. Poor, "Secret communication with feedback," International Symposium on Information Theory and Its Applications, ISITA 2008, pp. 1-6, 2008

[29] C. Li, Y. Liang, H. V. Poor, S. Shamai, "A coding scheme for colored Gaussian wiretap channels with feedback," 2018 IEEE International Symposium on Information Theory (ISIT), pp. 131-135, 2018

[30] J. P. M. Schalkwijk and T. Kailath, "A coding scheme for additive noise channels with feedback. part I: No bandwidth constraint," IEEE Trans. Inf. Theory, vol. 12, no. 2, pp. 172-182, February 1966.

[31] A. Wyner and J. Ziv, "The rate-distortion function for source coding with side information at the decoder," IEEE Trans. Inf. Theory, vol. 22, no. 1, pp. 1-10, January 1976.

[32] K. Marton, "A coding theorem for the discrete memoryless broadcast channel," IEEE Trans. Inf. Theory, vol. 25, no. 3, pp. 306-311, March 1979.
[33] A. El Gamal, Y. Kim, Network information theory. Cambridge University Press, 2011

[34] R. Timo, A. Grant, T. Chan, G. Kramer, "Source coding for a simple network with receiver side information," in Proc. Int. Symp. Inf. Theory, Toronto, Canada, Jul. 6-11, 2008, pp. 2307-2311

[35] B. Hajek, M. Pursley, "Evaluation of an achievable rate region for the broadcast channel," IEEE Trans. Inf. Theory, vol. 25, no. 1, pp. 36-46, January 1979.

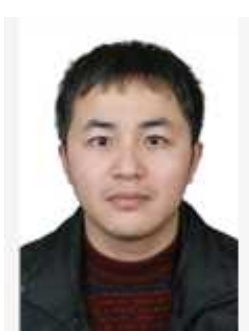

Bin Dai received the B.Sc. degree in Communications and Information systems from University of Electronic Science and Technology of China, Chengdu, China, in 2004. He received the M.Sc. and $\mathrm{Ph} . \mathrm{D}$. degrees in computer science and technology from Shanghai Jiaotong University, Shanghai, China, in 2007 and 2012, respectively. In 2011 and 2012, he was a Visiting Scholar with the Institute for Experimental Mathematics, Duisburg-Essen University, Essen, Germany. He is currently an Associate Professor with the Southwest Jiaotong University. His research interests include information-theoretic security, network information theory and coding.

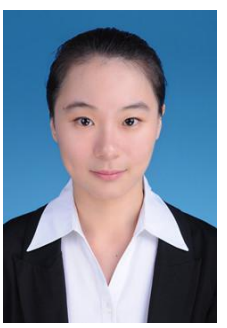

Linman Yu received the B.Sc. degree in economics and management from Sichuan Normal University, Chengdu, China, in 2012, and the M.Sc. degree in international economic and trade from the Dongbei University of Finance and Economics, Dalian, China, in 2014. She is currently a Lecturer with Chengdu Texile College. Her research interests include information theory and economics.

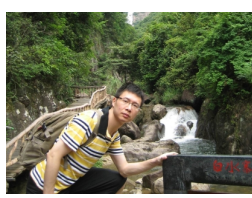

Xuxun Liu received the Ph.D. degree in communication and information system from Wuhan University, Wuhan, China, in 2007. He is currently an Associate Professor with the School of Electronic and Information Engineering, South China University of Technology, Guangzhou, China. He has authored or co-authored over 30 scientific papers in international journals and conference proceedings. His current research interests include wireless sensor networks, wireless communications, computational intelligence, and mobile computing.

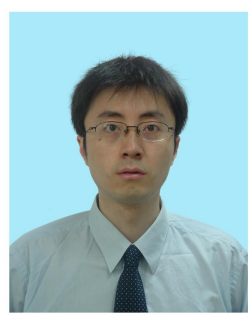

Zheng Ma received the B.Sc. and Ph.D. degrees in Communications and Information Systems from Southwest Jiaotong University, Chengdu, China, in 2000 and 2006, respectively. He was a Visiting Scholar with the University of Leeds, Leeds, U.K., in 2003. In 2003 and 2005, he was a Visiting Scholar with Hong Kong University of Science and Technology, Kowloon, Hong Kong. From 2008 to 2009, he was a Visiting Research Fellow with the Department of Communication Systems, Lancaster University, Lancaster, U.K. He is currently a Professor with the Southwest Jiaotong University. His research interests include information theory and coding, communication systems, signal design and processing, field-programmable gate array/digital signal processor implementation, and professional mobile radio. Dr. Ma has been the Vice-Chairman of the IT Chapter of the IEEE Chengdu Section since 2009. 